\title{
Sound-turbulence interaction in low Mach number duct flow
}

\author{
Chenyang Weng*, Susann Boij ${ }^{\dagger}$ \\ KTH Royal Institute of Technology, School of Engineering Sciences, \\ The Marcus Wallenberg Laboratory for Sound and Vibration, \\ Linn Flow Centre, Stockholm, Sweden \\ Ardeshir Hanifi ${ }^{\ddagger}$ \\ KTH Royal Institute of Technology, School of Engineering Sciences, \\ Department of Mechanics, \\ Linn Flow Centre, Stockholm, Sweden
}

\begin{abstract}
When sound waves propagate in ducts with the presence of turbulent flow, turbulent stresses can cause extra attenuation of the wave in addition to that caused by viscothermal effects. Different models for the turbulent stress acting on the sound waves are investigated in this study, and they are applied to compute the wall shear stress impedance of sound waves. Among these models the non-equilibrium model proposed by the present authors in another paper is studied in details. Attempt of including mean flow convection effect in the non-equilibrium model is made. The resulting modified model is then applied to compute the wave attenuation, and the results are compared with experimental data.
\end{abstract}

\section{Nomenclature}

\begin{tabular}{|c|c|c|c|}
\hline$A^{+}$ & Profile constant & $T$ & Temperature field, $\mathrm{K}$ \\
\hline$F^{\prime}$ & urbulent fluctuation quantity & $t$ & Time, s \\
\hline $\bar{F}$ & ean flow quantity & $t_{\tau}$ & Turbulent relaxation time scale, $\mathrm{s}$ \\
\hline$\tilde{F}$ & Perturbation field quantity & $u$ & ty field, $\mathrm{m} / \mathrm{s}$ \\
\hline $\boldsymbol{x}$ & $\begin{array}{l}\text { Cartesian coordinate position in vector } \\
\text { form, } \mathrm{m}\end{array}$ & $\begin{array}{l}u_{\tau} \\
x_{i} \\
u^{+}\end{array}$ & $\begin{array}{l}\text { Friction velocity, } \mathrm{m} / \mathrm{s} \\
\text { Cartesian coordinate position vector, } \mathrm{m}\end{array}$ \\
\hline $\begin{array}{l}\mathrm{He}=k_{0} R \\
\mathrm{M}\end{array}$ & $\begin{array}{l}\text { Helmholtz number } \\
\text { Mach number }\end{array}$ & $\begin{array}{l}y \\
Z_{\tau}\end{array}$ & $\begin{array}{l}\text { Dimensionless wall distance } \\
\text { Dimensionless wall shear stress }\end{array}$ \\
\hline $\operatorname{Pr}=\nu / \chi$ & Prandtl number & $z_{\tau}$ & $\begin{array}{l}\text { impedance } \\
\text { Wall shear stress impedance, } \mathrm{kg} /\left(\mathrm{m}^{2} \mathrm{~s}\right)\end{array}$ \\
\hline & $\begin{array}{l}\text { Perturbation heat flux, Km/s } \\
\text { Perturbation Reynolds stress, } \mathrm{m}^{2} / \mathrm{s}^{2}\end{array}$ & $z_{q}$ & $\begin{array}{l}\text { Wall heat conduction impedance, } \\
\mathrm{W} /\left(\mathrm{m}^{2} \mathrm{~K}\right)\end{array}$ \\
\hline$c_{0}$ & Isentropic speed of sound, $\mathrm{m}^{2} / \mathrm{s}$ & & \\
\hline$c_{p}$ & $\begin{array}{l}\text { Heat capacity at constant pressure, } \\
\mathrm{J} /(\mathrm{kg} \cdot \mathrm{K}) \\
\text { Hydraulic diameter, } \mathrm{m}\end{array}$ & $\begin{array}{l}\text { Subscripts } \\
+,-\end{array}$ & $\begin{array}{l}\text { Down- and up-stream propagation } \\
\text { waves }\end{array}$ \\
\hline & Frequency, $\mathrm{Hz}$ & 0 & Values are evaluated at the ambient \\
\hline$H_{0}^{(1)}, H_{1}^{(1)}$ & $\begin{array}{l}\text { Hankel functio } \\
\text { first order }\end{array}$ & & $\begin{array}{l}\text { state } \\
\text { Cartesian tensor component }\end{array}$ \\
\hline$k, k_{0}$ & Wave & $w$ & $s$ are evaluated at the wall \\
\hline$l_{\mathrm{vor}}, l_{\mathrm{ent}}$ & $\begin{array}{l}\text { Vorticity- and entropy modes boundary } \\
\text { layer thicknesses, m }\end{array}$ & $x, r$ & $\begin{array}{l}\text { Axial and radial components in cylindri- } \\
\text { cal coordinates }\end{array}$ \\
\hline$l_{m}$ & Prandtl mixing length, $\mathrm{m}$ & ac, vor, ent & Acoustic, vorticity, and entropy mode \\
\hline & Pressure field, $\mathrm{N} / \mathrm{m}^{2}$ & Symbols & \\
\hline & Radius of a pipe, $m$ & & \\
\hline
\end{tabular}

*PhD Student, Department of Aeronautical and Vehicle Engineering.

$\dagger$ Associate Professor, Department of Aeronautical and Vehicle Engineering.

${ }^{\ddagger}$ Adjunct Professor, Department of Mechanics. Also at Swedish Defence Research Agency, FOI, Stockholm, Sweden 


\begin{tabular}{|c|c|c|c|}
\hline$\alpha$ & Attenuation coefficient, $\mathrm{Np} / \mathrm{m}$ & $\nu$ & Kinematic viscosity, $\mathrm{m}^{2} / \mathrm{s}$ \\
\hline$\chi$ & Thermal diffusivity, $\mathrm{m}^{2} / \mathrm{s}$ & $\omega$ & Angular frequency, rad/s \\
\hline$\delta_{A}^{+}$ & Dimensionless acoustic boundary layer & $\rho$ & Density, $\mathrm{kg} / \mathrm{m}^{3}$ \\
\hline & thickness & $\tau_{w}$ & Mean flow wall shear stress, $\mathrm{kg} /\left(\mathrm{ms}^{2}\right)$ \\
\hline$\epsilon_{i j k}$ & Permutation tensor & \multirow{2}{*}{\multicolumn{2}{|c|}{ Superscripts }} \\
\hline$\kappa$ & The von Karman constant & & \\
\hline$\kappa_{\text {th }}$ & Thermal conductivity, W/(mK) & + & Dimensionless quantities that normal- \\
\hline$\mu$ & Dynamic viscosity, kg/(ms) & & ized by wall parameters \\
\hline
\end{tabular}

\section{Introduction}

\section{I.A. Background}

When sound waves propagate in turbulent duct flows, the acoustic and turbulent boundary layers exist simultaneously in the vicinity of the duct wall. In the case of low Mach number flows ${ }^{\S}$, the sound wave field can be represented as a superposition of acoustic, vorticity, and entropy modal wave fields ${ }^{1}$. For wave particle velocity, this can be written as

$$
\tilde{u}_{i}=\tilde{u}_{\mathrm{ac}, i}+\tilde{u}_{\mathrm{vor}, i}+\tilde{u}_{\mathrm{ent}, i} .
$$

The generation of the vorticity and the entropy modes is related to viscous and thermal diffusion respectively near the wall, and the amplitudes of these two modes decay away from the wall by a factor of $e$ within the corresponding boundary layer thicknesses $l_{\text {vor }}$ and $l_{\text {ent }}$, defined by

$$
l_{\mathrm{vor}}=\sqrt{\frac{2 \nu}{\omega}}, \quad l_{\mathrm{ent}}=\sqrt{\frac{2 \chi}{\omega}}=\frac{l_{\mathrm{vor}}}{\sqrt{\mathrm{Pr}}} .
$$

The Prandtl number Pr is assumed to be constant and of the order of one in air, so $l_{\text {vor }} \approx l_{\text {ent }}$, and $l_{\text {vor }}$ is usually referred to as "acoustic boundary layer thickness". The region outside the acoustic boundary layer is dominated by the acoustic mode.

For a given turbulent flow, Eq. (2) shows that vorticity and entropy modes diffuse faster (smaller boundary layer thickness) from the duct wall to the edge of the acoustic boundary layer at high frequencies. In these cases, $l_{\text {vor }}$ is essentially smaller than the turbulent viscous sublayer thickness $\left(\approx 5 \nu / u_{\tau}\right)$, so the vorticity and the entropy modes die out before they undergo turbulent diffusions.

However, at sufficiently low frequencies, these two modes penetrate into the turbulent buffer layer, and even the log-law region. This means that the propagation of the vorticity and the entropy modes is strongly influenced by the turbulent mixing of momentum and heat respectively, leading to extra sound attenuation besides that caused by viscothermal effects. The wall shear stress impedance and the wall heat conduction impedance, defined by ${ }^{2}$

$$
z_{\tau}=\left(\frac{\mu \partial \tilde{u}_{\mathrm{vor}, 1} / \partial x_{2}}{\tilde{u}_{\mathrm{vor}, 1}}\right)_{w}, \quad z_{q}=\left(\frac{\kappa_{\mathrm{th}} \partial \tilde{T}_{\mathrm{ent}} / \partial x_{2}}{\tilde{T}_{\mathrm{ent}}}\right)_{w},
$$

are inevitably affected by the turbulence as well. Here $x_{1}$ and $x_{2}$ denote directions tangential and normal to the wall, respectively.

From the above analysis, one can deduce that at low Mach numbers the effects of turbulent mixing on sound propagation, and the wall impedance, depend on the parameter ${ }^{\pi}$

$$
\delta_{A}^{+}=\frac{l_{\mathrm{vor}} u_{\tau}}{\nu}=\sqrt{\frac{2 u_{\tau}^{2}}{\omega \nu}},
$$

i.e., the ratio of the thickness of the acoustic boundary layer and the turbulent viscous sublayer length scale. The wave attenuation is influenced by turbulent flows only when $\delta_{A}^{+} \gg 1$. Experiments ${ }^{2-4}$ show that

\footnotetext{
$\S$ It is not necessary to be low Mach number to apply the superposition principle. But here we discuss the simplified case where the effect of the mean flow velocity convection is negligible.

IThis assumes that effect of turbulent mixing on sound propagation is independent of the Reynolds number based on the diameter of the duct. In fact even if there exists any dependence on the Reynolds number, it is rather small and can be neglected. Please see Ronneberger and $\mathrm{Ahrens}^{2}$ for detailed discussion.
} 
turbulent absorption of acoustic energy becomes more dominant than the molecular effects when $\delta_{A}^{+}$is larger than approx. 12. The effects of turbulent diffusion of momentum on the wall shear stress impedance $z_{\tau}$ are also observed in measurement ${ }^{2}$ and Large-Eddy Simulation (LES). ${ }^{5}$

The purpose of this study is to investigate the mechanism of the turbulent diffusion effects on sound wave propagation. The study scope is limited to incompressible fully developed turbulent flows in duct with constant cross section and smooth walls. For the sound waves, the Helmholtz number, $\mathrm{He}=k_{0} R$, is much smaller than one so the wave length is much larger than the duct cross section hydraulic diameter $D$. This also ensures that only plane waves propagate in the duct"l. The scattering and generation of sound by turbulence are negligible at low Mach numbers, so only sound absorption by turbulence is considered.

Over the years many researchers have tried to tackle the same, or similar, problems, mostly focusing on the modeling of the Reynolds stress (turbulent stress) acting on the sound waves. A commonly used mathematical description of the turbulent flow with sound waves is the "triple decomposition": let $F\left(x_{i}, t\right)$ represent a flow quantity in the turbulent flow system with the presence of the sound wave, it is decomposed into three parts 6,7

$$
F\left(x_{i}, t\right)=\bar{F}\left(x_{i}\right)+F^{\prime}\left(x_{i}, t\right)+\widetilde{F}\left(x_{i}, t\right),
$$

where $\bar{F}$ is the mean or the ambient value, $F^{\prime}$ corresponds to the turbulent fluctuation, and $\widetilde{F}$ is the coherent sound wave perturbation. The mean contribution $\bar{F}$ could be calculated from the time average of $F(\boldsymbol{x}, t)$, given by

$$
\bar{F}(\boldsymbol{x})=\lim _{\mathcal{T} \rightarrow \infty} \frac{1}{\mathcal{T}} \int_{t}^{t+\mathcal{T}} F\left(\boldsymbol{x}, t^{\prime}\right) \mathrm{d} t^{\prime}
$$

If we assume a single frequency sound wave, the phase average will give both the mean and sound wave quantities, which is

$$
\langle F(\boldsymbol{x}, t)\rangle=\lim _{N \rightarrow \infty} \frac{1}{N} \sum_{n=0}^{N} F\left(\boldsymbol{x}, t+\frac{n}{f}\right),
$$

where $f$ is the frequency of the sound wave.

Using the definitions given in Eqs. (6) and (7), the Navier-Stokes equations can be reduced to a linear system of equations for harmonic perturbation in a turbulent flow, see Refs. 6-8. In these equations the Reynolds stress terms appear as**

$$
\tilde{r}_{i j}=\left\langle u_{i}^{\prime} u_{j}^{\prime}\right\rangle-\overline{u_{i}^{\prime} u_{j}^{\prime}},
$$

which is interpreted as the oscillation of the background Reynolds stress due to the organised wave perturbations, so herein we call $\tilde{r}_{i j}$ the perturbation Reynolds stress. To simplify the modeling of $\tilde{r}_{i j}$, we can assume that the amplitudes of the sound field quantities are very small, so the mean flow field is not affected by the presence of the sound waves. For the time averaged Reynolds stress $\overline{u_{i}^{\prime} u_{j}^{\prime}}$ in Eq. (8), we can therefore use available models for turbulent flows without sound waves. However, the phase averaged term, $\left\langle u_{i}^{\prime} u_{j}^{\prime}\right\rangle$, requires the modeling of the Reynolds stress which will now be modulated considering to the presence of the sound wave.

\section{I.B. Previous models for the perturbation Reynolds stress}

The perturbation Reynolds stress, (8), can be modeled with different levels of complexity. The simplest model is the quasi-laminar model. This model neglects the modulation of the Reynolds stress by assuming that the turbulent flow is entirely uninfluenced by the small amplitude wave perturbations, giving $\left\langle u_{i}^{\prime} u_{j}^{\prime}\right\rangle=\overline{u_{i}^{\prime} u_{j}^{\prime}}$ and $\tilde{r}_{i j}=0$. Based on what has been discussed earlier, the quasi-laminar model is supposed to be valid only when $\delta_{A}^{+}$is small, i.e., when the vorticity mode dies out within the turbulent viscous sublayer. This has been experimentally proved. ${ }^{2,3,6}$ Consequently, the modeling of the perturbation Reynolds stress at larger values of $\delta_{A}^{+}$requires the modulation of the Reynolds stress to be considered. So the sound waves are actually interacting with the turbulence, rather than merely being affected by the flows.

The so-called quasi-static model ${ }^{2,6,9-11}$ is an attempt to include the sound-turbulence interaction in the modeling of $\tilde{r}_{i j}$. It assumes the sound waves to represent a quasi-static modulation of the Reynolds

\footnotetext{
${ }$ For circular cross-section duct, if the sound wave frequency is smaller than the cutoff frequency for the first non-plane mode, the Helmholtz number is then smaller than 1.841. Please see, for example, Pierce ${ }^{1}$.

${ }^{* *}$ The stress is apparently $\rho_{0}\left(\left\langle u_{i}^{\prime} u_{j}^{\prime}\right\rangle-\overline{u_{i}^{\prime} u_{j}^{\prime}}\right)$, but the density is conveniently and conventionally omitted here.
} 
stress, i.e., still neglecting the non-equilibrium effects introduced by the wave oscillations on the turbulent flows. The Boussinesq turbulent viscosity hypothesis can therefore be applied to the modeling of $\left\langle u_{i}^{\prime} u_{j}^{\prime}\right\rangle$. Mathematically, this can be represented as ${ }^{2,11,12}$

$$
\left\langle u_{1}^{\prime} u_{2}^{\prime}\right\rangle=-l_{m}^{2}\left[\frac{\partial\left(\bar{u}_{1}+\tilde{u}_{1}\right)}{\partial x_{2}}\right]^{2}
$$

in a simple shear flow, where $l_{m}$ is the mixing length, which is assumed to be uninfluenced by the sound waves in the quasi-static assumption. This gives the expression ${ }^{\dagger \dagger}$ for $\tilde{r}_{12}$

$$
\tilde{r}_{12}=\left\langle u_{1}^{\prime} u_{2}^{\prime}\right\rangle-\overline{u_{1}^{\prime} u_{2}^{\prime}} \approx-2 \nu_{T} \frac{\partial \tilde{u}_{1}}{\partial x_{2}},
$$

where

$$
\nu_{T}=l_{m}^{2}\left|\frac{\partial \bar{u}_{1}}{\partial x_{2}}\right|
$$

is the equilibrium eddy viscosity, and the linear approximation is made in the derivation of Eq. (10). This quasi-static approximation is more physically correct than the quasi-laminar model, and experiments have shown that it can qualitatively predict the turbulent diffusion effects at larger values of $\delta_{A}^{+}$.

At $\delta_{A}^{+} \approx 12$, however, the measured sound attenuation and the real part of the wall shear stress impedance display a local minimum value, which can not be predicted by the quasi-static model. ${ }^{2,3,11}$ Several attempts have been made to model this local minimum. By neglecting the eddy viscosity within the viscous sublayer, equaled to $7 \nu / u_{\tau}$, while using an eddy viscosity linearly dependant on the distance from the wall, Howe ${ }^{11}$ predicted a local minimum. Ronneberger and Ahrens ${ }^{2}$ also assumed that the eddy viscosity is zero from the wall to the edge of the viscous sublayer $\left(=15 \nu / u_{\tau}\right)$, then the turbulence is modeled as a rigid plate at the edge of the sublayer to reflect the wave. This rigid plate model shows qualitative agreement with the measurement, however the local minimum is predicted but at incorrect value of $\delta_{A}^{+}$. Peters $e t$ al. ${ }^{3}$ and Howe ${ }^{13}$ later implicitly included the non-equilibrium effect in the rigid plate model and the model proposed by Howe, ${ }^{11}$ respectively, and the predicted results are much improved. Mao and Hanratty ${ }^{14}$ also introduced a relaxation model to include the non-equilibrium effect in the mixing length $l_{m}$ by relaxing the oscillation pressure gradient, and the predicted result also shows better agreement with the measurements than the quasi-static model. This stresses the important role of the non-equilibrium effect in the sound-turbulent interaction, where neither the quasi-laminar nor the quasi-static assumption is sufficient to describe the interaction at intermediate values of $\delta_{A}^{+}$. Nevertheless, the model introduced by Howe, ${ }^{13}$ including some succeeding modified versions, ${ }^{15,16}$ and the relaxation model proposed by Mao and Hanratty ${ }^{14}$ are based on empirical formulas; the rigid plate models introduced by Ronneberger and Ahrens ${ }^{2}$ and Peters et al. ${ }^{3}$ are merely rough approximations. So a more physical model is needed to understand and model the mechanism of the non-equilibrium interaction. For this reason, the present authors recently proposed a non-equilibrium model ${ }^{17}$ for $\tilde{r}_{i j}$, which is directly derived from the Reynolds stress equations for $\overline{u_{i}^{\prime} u_{j}^{\prime}}$ and $\left\langle u_{i}^{\prime} u_{j}^{\prime}\right\rangle$. Unlike the quasi-static assumption, this model shows that the turbulent flow acts as a viscoelastic fluid, instead of purely viscous, to the sound wave during the interaction process, i.e., the perturbation turbulent stress $\tilde{r}_{i j}$ does not only depend on the instantaneous sound wave shear strain rate, but also has the "memory" of its past.

In this paper, wave attenuations and wall shear stress impedance are computed by using this new model, the predicted results are compared with experiments, as well as some previous models. Satisfactory agreements between the new model and the measurements of both the wave attenuations and wall shear stress impedance are observed. In addition, an attempt is made to include mean flow convection effects in this new model.

\footnotetext{
${ }^{\dagger \dagger}$ By assuming the turbulent kinetic energy is not modulated by the wave motion, Reynolds and Hussain ${ }^{6}$ have modelled $\tilde{r}_{i j}$ as half that in Eq. (10).
} 


\section{Modelling of acoustic boundary layer problems}

\section{II.A. Summary of acoustic boundary layer theory for cases when turbulent diffusion effects are neglected}

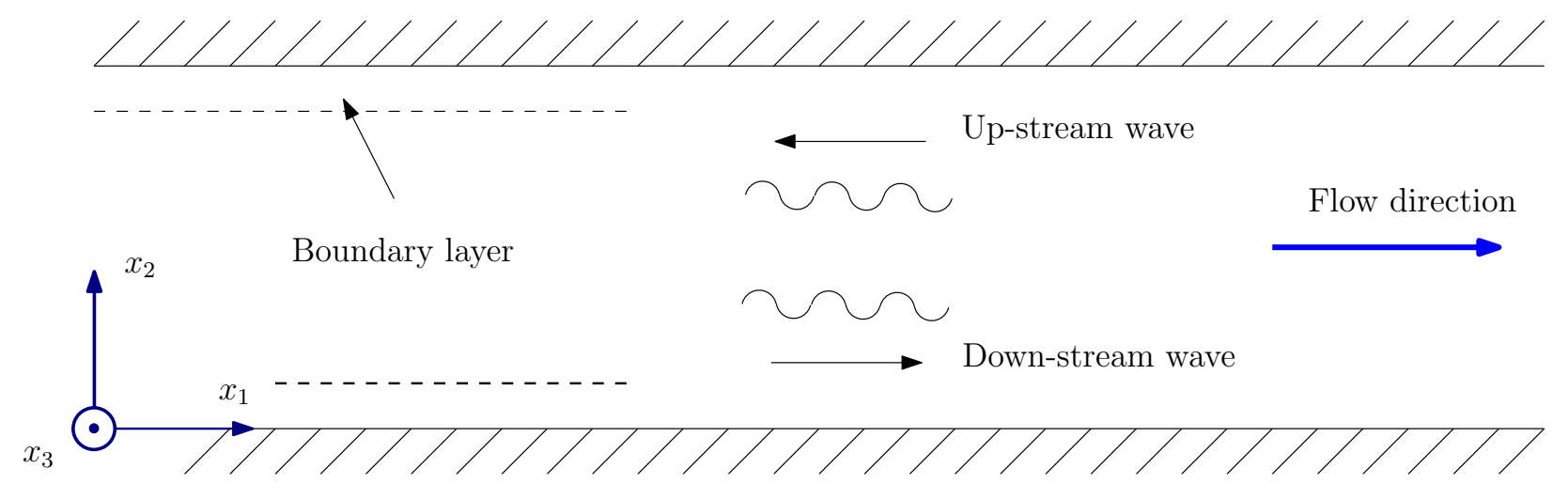

Figure 1. Cartesian coordinate system used to describe the flow and sound fields in the duct. Note that the thickness of the boundary layer (both for acoustic and turbulent) in the sketch is exaggerated compared to the real situation studied here.

To begin with, we discuss the cases when the acoustic boundary layer thickness $l_{\text {vor }}$ is much smaller than the turbulent viscous sublayer $\left(\delta_{A}^{+} \ll 1\right)$, so turbulent diffusion can be neglected. For such cases one can solve the linearized perturbation equations, e.g. as derived from the Navier-Stokes equations, see Kierkegaard et $a l .,{ }^{18}$ to obtain numerical solutions for the sound fields. Analytical solutions, however, are usually difficult to get from these coupled equations, but based on the whole linearized equations themselves one can analyze the dynamics of the system. To have a deeper insight into such an acoustic boundary layer problem, a mode superposition method can be applied to the perturbation equations, and three much simplified uncoupled equations can be derived for three decomposed modes respectively, i.e., the acoustic, vorticity, and entropy modes. Analytical solutions are then available to analyze the system.

We herein assume a two dimensional plane sound wave with time dependency in the form exp (i $\omega t$ ), and the wavelength of the sound wave to be much larger than the duct hydraulic diameter. Besides, the duct is assumed to be wide enough that the viscothermal acoustic boundary layers, $l_{\text {vor }}$ and $l_{\text {ent }}$, are much smaller than the wall hydraulic diameter, so the vorticity and entropy modes vary more rapidly in the $x_{2}$ direction compared to that in the $x_{1}$ direction (see Fig. 1). In addition, the Mach number of the flow is small, i.e., $\mathrm{M} \ll 1$, so the convection by mean flow velocity is negligible. For an ideal gas, the equations for the three modes, and the corresponding polarization relations, in a two dimension approximation $\left(\partial / \partial x_{3}=0\right)$ can be then written as ${ }^{1}$

Acoustic mode ${ }^{\ddagger \ddagger}$

$$
\begin{gathered}
\left(\frac{\partial^{2}}{\partial x_{i} \partial x_{i}}+\frac{\omega^{2}}{c_{0}}\right) \tilde{p}_{\mathrm{ac}}=0 \\
\epsilon_{i j k} \frac{\partial \tilde{u}_{k, \mathrm{ac}}}{\partial x_{j}}=0, \quad \tilde{s}_{\mathrm{ac}} \approx 0, \quad \mathrm{i} \rho_{0} \omega \tilde{u}_{\mathrm{ac}, i} \approx-\frac{\partial \tilde{p}_{\mathrm{ac}}}{\partial x_{i}} \\
\tilde{T}_{\mathrm{ac}} \approx \frac{1}{\rho_{0} c_{p}} \tilde{p}_{\mathrm{ac}}, \quad \tilde{\rho}_{\mathrm{ac}} \approx \frac{1}{c_{0}^{2}} \tilde{p}_{\mathrm{ac}} .
\end{gathered}
$$

Vorticity mode

$$
\begin{gathered}
\left(\frac{\partial^{2}}{\partial x_{2} \partial x_{2}}-\frac{\mathrm{i} \omega}{\nu}\right) \tilde{u}_{\mathrm{vor}, i}=0 \\
\frac{\partial \tilde{u}_{\mathrm{vor}, i}}{\partial x_{i}}=0, \quad \tilde{p}_{\mathrm{vor}}=\tilde{s}_{\mathrm{vor}}=\tilde{T}_{\mathrm{vor}}=\tilde{\rho}_{\mathrm{vor}}=0 .
\end{gathered}
$$

\footnotetext{
$\ddagger \ddagger$ The acoustic mode equation Eq. (12) should also include the viscothermal and relaxation absorptions of the fluid. But they are much smaller than the absorption in the boundary layer for our investigation, hence omitted here.
} 


\section{Entropy mode}

$$
\begin{gathered}
\left(\frac{\partial^{2}}{\partial x_{2} \partial x_{2}}-\frac{\mathrm{i} \omega}{\chi}\right) \tilde{s}_{\mathrm{ent}}=0 \\
\epsilon_{i j k} \frac{\partial \tilde{u}_{k, \mathrm{ent}}}{\partial x_{j}}=0, \quad \tilde{p}_{\mathrm{ent}} \approx 0, \quad \tilde{u}_{\mathrm{ent}, i} \approx \frac{\chi}{c_{p}} \frac{\partial \tilde{s}_{\mathrm{ent}}}{\partial x_{i}}, \\
\tilde{T}_{\mathrm{ent}} \approx \frac{T_{0}}{c_{p}} \tilde{s}_{\mathrm{ent}}, \quad \tilde{\rho}_{\mathrm{ent}} \approx \frac{\rho_{0}}{c_{p}} \tilde{s}_{\mathrm{ent}} .
\end{gathered}
$$

The approximately equal signs in the polarization equations Eqs. (13) and (17) are due to the "long" wavelength (compared to duct hydraulic diameter $D$ ) assumption made earlier, and they will be replaced by equal signs in the following text, since this assumption is well satisfied in all the experiments that will be mentioned in this study.

One recognizes that the acoustic mode is the solution to the perturbation equations in isentropic and ideal fluid, and it occupies almost the entire area of a duct cross-section if the boundary layer is thin. The governing equations for the vorticity and entropy modes, Eq. (14) and (16), are viscous- and thermal-diffusion equations respectively, which have the following solutions

$$
\tilde{u}_{\mathrm{vor}, i}=\tilde{u}_{\mathrm{vor}, i, w} e^{-(1+\mathrm{i}) x_{2} / l_{\mathrm{vor}}}, \quad \tilde{s}_{\mathrm{ent}}=\tilde{s}_{\mathrm{ent}, w} e^{-(1+\mathrm{i}) x_{2} / l_{\mathrm{ent}}},
$$

where $l_{\text {vor }}$ and $l_{\text {ent }}$ are defined in Eq. (2). Inserting the solution for $\tilde{s}_{\text {ent }}$ into Eq. (17) to calculated the $x_{1}$ component of the entropy mode velocity $\tilde{u}_{\text {ent, } 1 \text { yields }}$

$$
\tilde{u}_{\text {ent }, 1}=\frac{\chi}{c_{p}} \frac{\partial \tilde{s}_{\text {ent }}}{\partial x_{1}}=0
$$

and this is in accord with the "long" wavelength assumption $\left|\partial \tilde{s}_{\text {ent }} / \partial x_{1}\right| \ll\left|\tilde{s}_{\text {ent }}\right| / l_{\text {ent }}$.

On the duct wall, if we apply the no-slip $\left(\tilde{u}_{1, w}=\tilde{u}_{\mathrm{ac}, 1, w}+\tilde{u}_{\mathrm{vor}, 1, w}=0\right)$ and iso-thermal $\left(\tilde{T}=\tilde{T}_{\mathrm{ac}, w}+\right.$ $\tilde{T}_{\text {ent }, w}=0$ ) boundary conditions, we can have the following relation derived from Eqs (13), (17) and (18),

$$
\tilde{u}_{\mathrm{vor}, 1}=-\tilde{u}_{\mathrm{ac}, 1} e^{-(1+\mathrm{i}) x_{2} / l_{\mathrm{vor}}}
$$

and

$$
\tilde{T}_{\text {ent }}=-\frac{1}{\rho_{0} c_{p}} \tilde{p}_{\text {ac }} e^{-(1+\mathrm{i}) x_{2} / l_{\text {ent }}}
$$

where the acoustic mode variables $\tilde{u}_{\mathrm{ac}, 1}$ and $\tilde{p}_{\text {ac }}$ are assumed to vary insignificantly along the $x_{2}$ direction. Eqs. (20) and (21) show that the vorticity and entropy mode can be determined from the acoustic modal fields. Here one should notice that it is not the individual mode, but the superposition of the three modes that satisfy the boundary conditions.

For the waves outside the thin acoustic boundary layers, a plane wave solution takes the form

$$
\tilde{p}=\hat{\tilde{p}} e^{\mathrm{i}\left(\omega t-k x_{1}\right)} .
$$

Due to the existence of the acoustic boundary layers, the acoustic energy is dissipated during the propagation. So, instead of being a real number $\omega / c_{0}$ in the case of propagation in an ideal fluid, the wavenumber $k$ is a complex number in this case. The imaginary part of $k$, i.e., the attenuation coefficient $\alpha$, represents the dissipations due to the viscous- and thermal-diffusions in the boundary layer, while the real part can be used to calculate the phase velocity changes. The classical Kirchhoff solution for $k$ for wide pipes is ${ }^{1,19}$

$$
k=\frac{\omega}{c_{0}}-\frac{1+\mathrm{i}}{l_{\text {vor }}} \frac{2 \mathrm{i} \nu}{D c_{0}}\left(1+\frac{\gamma-1}{\sqrt{\mathrm{Pr}}}\right),
$$

where $\operatorname{Pr}=\nu / \chi$ is assumed. Note that Eq. (3) and (18) give

$$
\frac{1+\mathrm{i}}{l_{\mathrm{vor}}}=\frac{-1}{\tilde{u}_{\mathrm{vor}, 1, w}}\left(\frac{\partial \tilde{u}_{\mathrm{vor}, 1}}{\partial x_{2}}\right)_{w}=\frac{-z_{\tau}}{\rho_{0} \nu},
$$


so Eq. (23) becomes

$$
k=\frac{\omega}{c_{0}}+\frac{2 \mathrm{i} z_{\tau}}{\rho_{0} c_{0} D}\left(1+\frac{\gamma-1}{\sqrt{\mathrm{Pr}}}\right) .
$$

Eq. (25) relates the attenuation of the sound waves to the wall shear stress impedance $z_{\tau}$ for low Mach number flows. The real part of $z_{\tau}$ relates to the attenuation of the wave, while the imaginary part relates to the phase shift. Since $z_{\tau}$ is computed from the vorticity field, which, as shown by (15), corresponds to an incompressible flow, we can therefore estimate the effect of shear stress forces (including the Reynolds stress) on the wave propagation from the incompressible flow calculations.

\section{II.B. Influence of turbulent diffusions}

As has been discussed earlier, at large values of the non-dimensional acoustic boundary layer thickness, $\delta_{A}^{+}$, the vorticity and entropy modes are influences by turbulent stresses and heat transfers, respectively. These effects can be included by modifying Eqs. (14) and (16). We herein assume that the turbulent heat flux can be related to the Reynolds stress by, for example, the gradient-diffusion hypotheses ${ }^{12}$ or the gradient diffusion model, ${ }^{20}$ so only the turbulent stress modeling is focused on here.

By using the triple decomposition and the phase/time averages, as defined in Eqs (5) to (7), the vorticity equation for the $x_{1}$ component can be rewritten as ${ }^{6,17,21}$

$$
\nu \frac{\partial^{2} \tilde{u}_{\mathrm{vor}, 1}}{\partial x_{2} \partial x_{2}}-\frac{\partial \tilde{r}_{12}}{\partial x_{2}}-\mathrm{i} \omega \tilde{u}_{\mathrm{vor}, 1}=0
$$

where $\tilde{r}_{i j}$, as defined in Eq. (8), acts as stresses on the vorticity mode. Since the phase average retain the contributions from both the mean flow and the sound wave, the differences $\left\langle u_{i}^{\prime} u_{j}^{\prime}\right\rangle-\overline{u_{i}^{\prime} u_{j}^{\prime}}$ will give the wave contribution to the Reynolds stress. Intrinsically one can relate $\tilde{r}_{i j}$ to the wave shear strain rate, $\partial \tilde{u}_{\mathrm{vor}, 1} / \partial x_{1}$, via an eddy-viscosity $\nu_{T}$. This is the so-called quasi-static model, represented by Eq. (10). In the mixing length theory $\nu_{T}$ is defined by Eq. (11), while in practice $\nu_{T}$ is usually computed numerically from some empirical models, like the $\mathrm{k}-\epsilon$ and $\mathrm{k}-\omega$ models (see, for example, Pope, ${ }^{12}$ for more details). Herein we employ an empirical formula for $\nu_{T}$, as originally suggested by Cess. ${ }^{22}$ It is a combination of van Driest's wall region law and Reichadt's middle law, and it has the merit of being differentiable along the whole cross-section of the duct. The expression is ${ }^{23,24}$

$$
\nu_{T}^{+}=\frac{\nu_{T}}{\nu}=\frac{1}{2} \sqrt{1+\frac{\kappa^{2} \mathrm{Re}_{\tau}^{2}}{9}\left[1-\left(\frac{r^{+}}{\operatorname{Re}_{\tau}}\right)^{2}\right]^{2}\left[1+2\left(\frac{r^{+}}{\operatorname{Re}_{\tau}}\right)^{2}\right]^{2}\left(1-e^{-y^{+} / A^{+}}\right)^{2}}-\frac{1}{2},
$$

where $\operatorname{Re}_{\tau}$ is the friction Reynolds number, and for pipe flow it is $\operatorname{Re}_{\tau}=u_{\tau} R / \nu$, and $y^{+}$is the dimensionless wall distance, which for pipe flow is defined by $y^{+}=(R-r) u_{\tau} / \nu=\operatorname{Re}_{\tau}-r^{+}$. The non-dimensional radius is defined by $r^{+}=r u^{\tau} / \nu$. Updated values ${ }^{25}$ for the von Karman constant $\kappa=0.42$ and the empirical constant $A^{+}=27$ are used in the calculation.

The van Driest's wall region law is accurate enough in the wall region; ${ }^{12}$ and for the outer region, Fig. 2 shows that the performance of this empirical model, Eq. (27), is also satisfactory by comparing to the experimental data ${ }^{\S \S}$ measured in fully developed turbulent pipe flows by McKeon et al.. ${ }^{26}$

Replacing the term $\nu_{T}$ in Eq. (10) with Cess' model, Eq. (27), we can compute the wall shear stress impedance $z_{\tau}$ from Eq. (26) for the limit when the Mach number tends to zero. The equation can be solved iteratively by e.g. the shooting method. In the following we use an alternative method to simplify the computation: note the derived result Eq. (19) gives $\tilde{u}_{\mathrm{vor}, 1}=\tilde{u}_{1}-\tilde{u}_{\mathrm{ac}, 1}$, and $\tilde{u}_{\mathrm{ac}, 1}$ can be related to $\mathrm{d} \tilde{p}_{\mathrm{ac}} / \mathrm{d} x_{1}$, according to the polarization relation in Eq. (13). We can therefore rewrite Eq. (26) as a perturbation equation for $\tilde{u}_{1}$. Since we will compare the computed results to pipe flow measurement, it is more convenient to write this perturbation equation in cylindrical coordinates, and the non-dimensional form of it is

$$
\left[\frac{1}{r^{+}} \frac{\partial}{\partial r^{+}}\left(r^{+} \nu_{e}^{+} \frac{\partial}{\partial r^{+}}\right)-\mathrm{i} \omega^{+}\right] \tilde{u}_{x}^{+}=\frac{\mathrm{d} \tilde{p}_{\mathrm{ac}}^{+}}{\mathrm{d} x^{+}}
$$

\footnotetext{
$\S \S$ The measured data of the mean velocity are not given in the paper by McKeon et al., ${ }^{26}$ but available from the online database "Superpipe Data" (http://www.princeton.edu/mae/people/faculty/smits/homepage/data-1/superpipe-data/mckeon/).
} 
where

$$
\left\{\begin{array}{l}
x^{+}=\frac{x u_{\tau}}{\nu}, \nu_{e}^{+}=1+2 \nu_{T}^{+} \\
\tilde{u}_{x}^{+}=\frac{\tilde{u}_{x}}{u_{\tau}}, \tilde{p}_{\mathrm{ac}}^{+}=\frac{\tilde{p}_{\mathrm{ac}}}{\rho_{0} u_{\tau}^{2}}, \omega^{+}=\frac{\omega \nu}{u_{\tau^{2}}}=\frac{2}{\delta_{A}^{+2}}
\end{array},\right.
$$

and the subscript $x$ here denotes the axial distance in the cylindrical coordinate. Here it is assumed that the variation of $\tilde{u}_{x}$ in the stream-wise direction is very small compared to that in the normal direction.

(a): Reynolds number: 144580

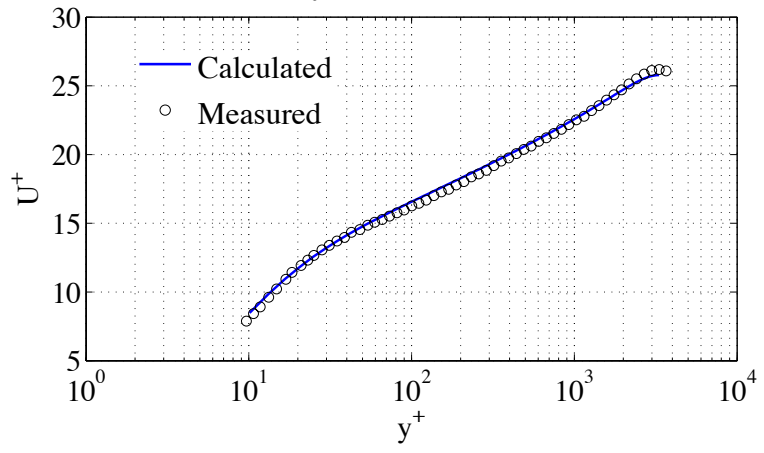

(c): Reynolds number: 309630

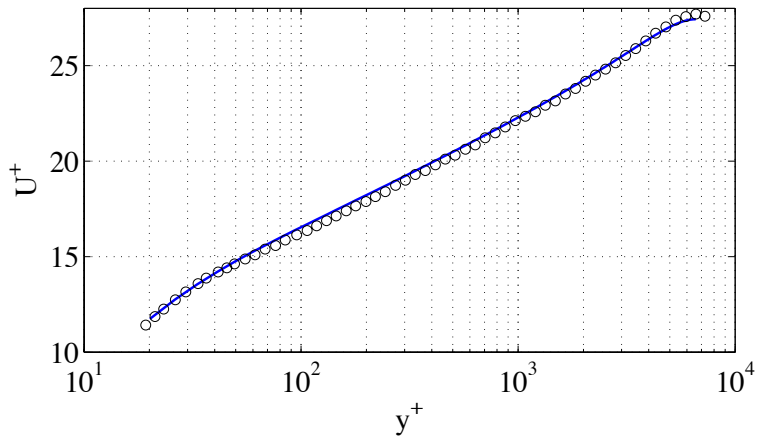

(e): Reynolds number: 536930

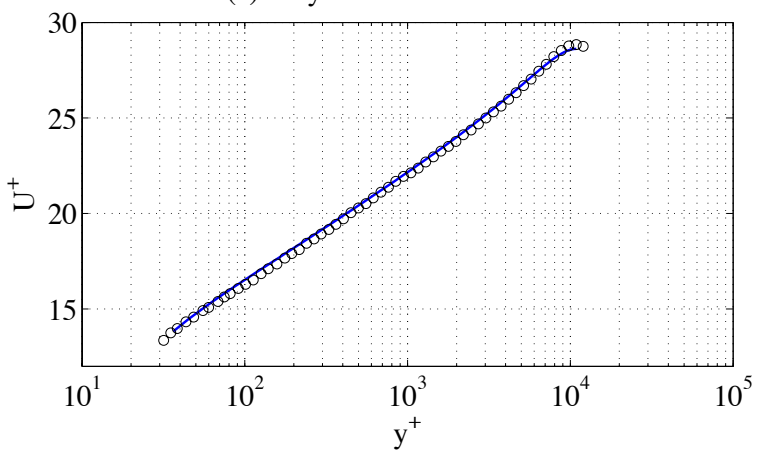

(b): Reynolds number: 233970

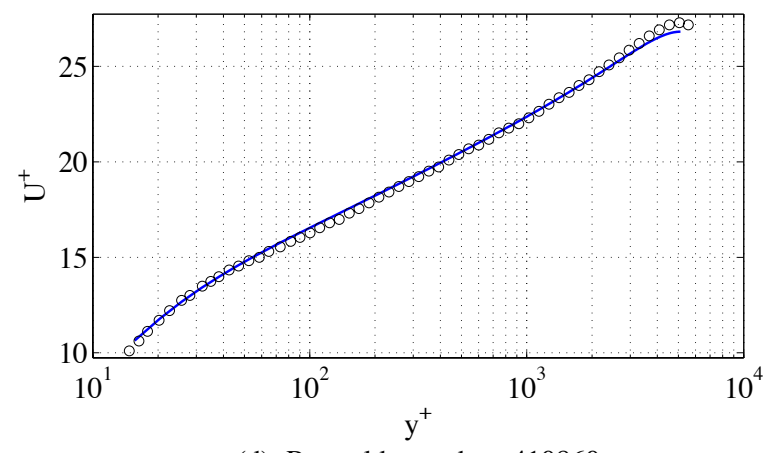

(d): Reynolds number: 410860

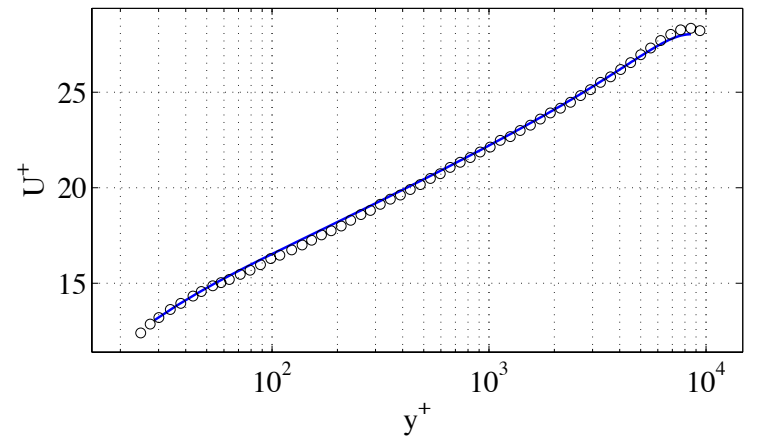

(f): Reynolds number: 74345

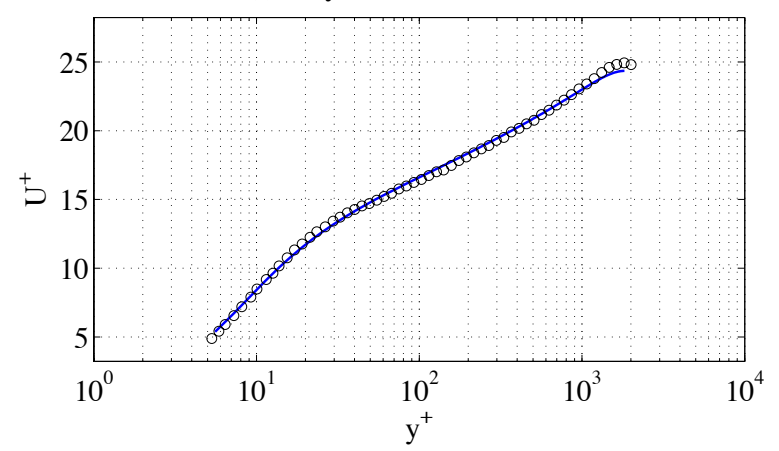

Figure 2. Comparisons of the turbulent mean velocity profiles in fully developed turbulent pipe flow computed with Eq. (27) and the experimental data measured by McKeon et al. ${ }^{26}$ The non-dimensional velocity is defined by $U^{+}=\bar{u}_{1} / u_{\tau}$; the Reynolds number is based on the bulk velocity of the mean flow and the pipe diameter.

Equation (28) is solved numerically by spectral method (see, for example, Boyd ${ }^{27}$ ) for different values of $\delta_{A}^{+}$. By setting $\mathrm{d} \tilde{p}_{\mathrm{ac}}^{+} / \mathrm{d} x^{+}=\mathrm{i} \sqrt{2 \omega^{+}}$for each calculation, the normalized wall shear stress impedance is determined by

$$
Z_{\tau}=\frac{z_{\tau}}{-\rho_{0} \sqrt{\omega \nu / 2}}=\left(\frac{\partial \tilde{u}_{x}^{+}}{\partial r^{+}}\right)_{w}
$$

From Eq. (20) we can see that $Z_{\tau}=1+\mathrm{i}$ in the quasi-laminar state, i.e. when turbulent diffusion is neglected. 
The computed values for $Z_{\tau}$ from Eq. (30), with the quasi-static model Eq. (10), are plotted in Fig. (3) by varying the non-dimensional acoustic boundary layer thickness $\delta_{A}^{+}$. Note that Cess' formula Eq. (27) depends on the friction Reynolds number $\mathrm{Re}_{\tau}$, which needs to be large enough as $\delta_{A}^{+}$increases, in order to satisfy the assumption that $l_{\text {vor }} / D \ll 1$. This is because $\delta_{A}^{+}=l_{\text {vor }} u_{\tau} / \nu=2 \operatorname{Re}_{\tau}\left(l_{\text {vor }} / D\right)$. In this paper the numerical value for $\operatorname{Re}_{\tau}$ is set to be 4000 to obtain converged results.

As has been discussed earlier in the introduction section, when the acoustic boundary thickness $l_{\text {vor }}$ is much larger than the turbulent viscous sublayer thickness $\left(\approx 5 \nu / u_{\tau}\right)$, turbulent diffusion of momentum will contribute to the sound attenuation. This indicates that the real part of $Z_{\tau}$ should be larger than unity when $\delta_{A}^{+}>5$. Fig. (3) shows that the quasi-static model is able to describe such an extra damping caused by the turbulent absorption. However, the experimental data shown in the same figure suggest a local minimum is experienced by the sound attenuation at $\delta_{A}^{+} \approx 12$, which is completely unpredicted by the quasi-static model.
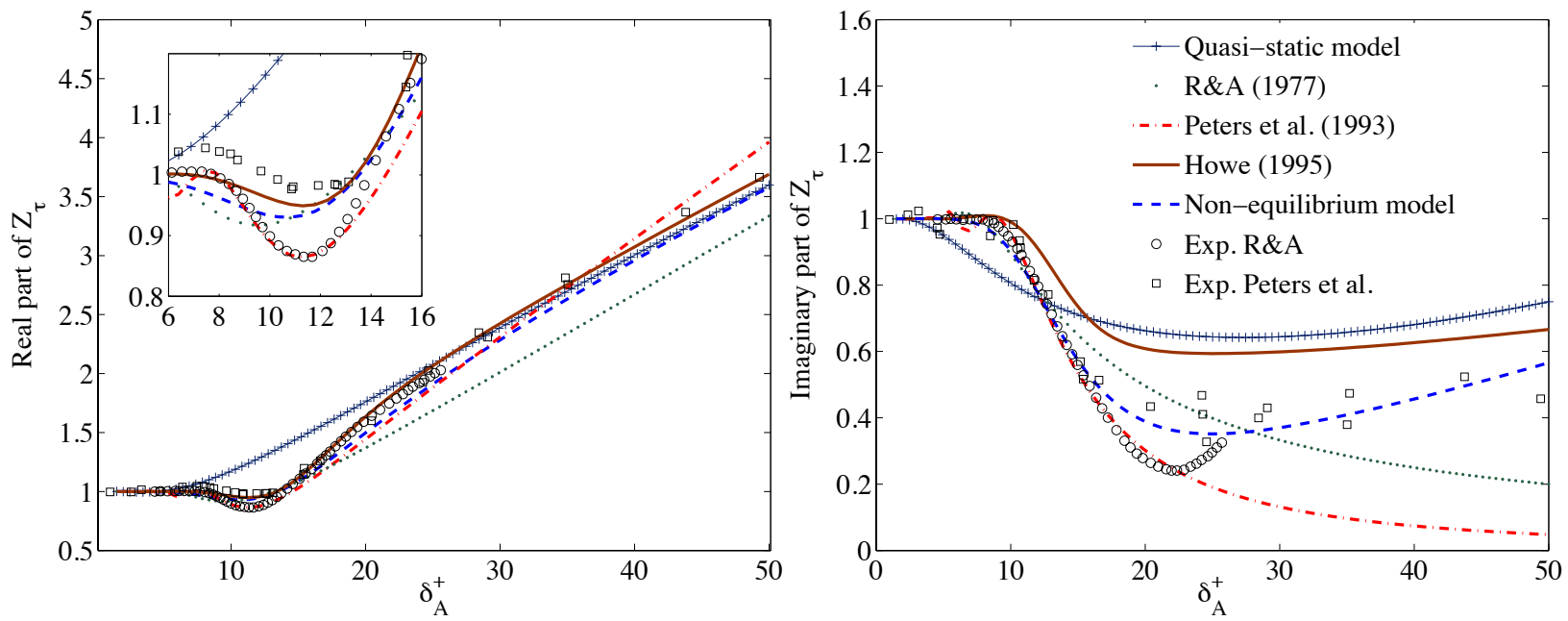

Figure 3. Computed real and imaginary parts of the normalized wall impedance of the vorticity mode, defined by Eq. (30), from different models, and the experimental data measured by Ronneberger and Ahrens (R\&A) ${ }^{2}$ and by Peters et al.. "Quasi-static model": computed from Eq. (28) by using Cess' eddy-viscosity formula Eq. (27), where $\mathbf{R e}_{\tau}=4000$; "R\&A (1977)": Eq. (32), $\phi=0$ and $\delta_{\nu}^{+}=15$; "Peters et al. (1993)": Eq. (32), $\phi=100 \omega^{+}$and $\delta_{\nu}^{+}=12.5$; "Howe (1995)": Eq. (35). "Non-equilibrium model" computed from Eq. (28) by using the perturbation eddy-viscosity in $\nu_{e}^{+}$, i.e., Eq. (40).

Ronneberger and Ahrens ${ }^{2}$ attributed this local minimum to the result of a destructive interference between the vorticity modal field that propagates from the wall and the wave which is reflected by the turbulence at the edge of the viscous sublayer. In a rough approximation, Ronneberger and Ahrens ${ }^{2}$ modelled the turbulence as a rigid plate placed at a distance $\delta_{\nu}$ from the wall, where $\delta_{\nu}$ corresponds to the thickness of the viscous sublayer. The boundary condition at $x_{2}=0$ is $\tilde{u}_{\mathrm{vor}, 1}(0)=\tilde{u}_{\mathrm{vor}, 1, w}$; at $x_{2}=\delta_{\nu}$, it is assumed that the wave reflected by the plate has a phase shift $\phi$, and for a totally reflected wave, i.e. $\tilde{u}_{\mathrm{vor}, 1}\left(\delta_{\nu}\right)=0$, the phase shift should be zero.

Applying this assumption in the vorticity mode equation, Eq. (14), we can get (in Cartesian coordinates)

$$
\frac{\tilde{u}_{\mathrm{vor}, 1}}{\tilde{u}_{\mathrm{vor}, 1, w}}=\cosh \left(y^{+} \sqrt{\mathrm{i} \omega^{+}}\right)-\frac{\cosh \left(\delta_{\nu}^{+} \sqrt{\mathrm{i} \omega^{+}}+\mathrm{i} \phi / 2\right)}{\sinh \left(\delta_{\nu}^{+} \sqrt{\mathrm{i} \omega^{+}}\right)} e^{-\mathrm{i} \phi / 2} \sinh \left(y^{+} \sqrt{\mathrm{i} \omega^{+}}\right),
$$

where $y^{+}=x_{2} u_{\tau} / \nu, \delta_{\nu}^{+}=\delta_{\nu} u_{\tau} / \nu$. Eq. (31) allows us to compute the wall shear stress impedance as

$$
Z_{\tau}=(1+\mathrm{i}) \frac{\cosh \left(\delta_{\nu}^{+} \sqrt{\mathrm{i} \omega^{+}}+\mathrm{i} \phi / 2\right)}{\sinh \left(\delta_{\nu}^{+} \sqrt{\mathrm{i} \omega^{+}}\right)} e^{-\mathrm{i} \phi / 2} .
$$

In Ronneberger and Ahrens' ${ }^{2}$ original rigid plate model, the vorticity mode is assumed to be totally reflected, corresponding to $\phi=0 ; \delta_{\nu}^{+}=15$ is chosen to fit the their measured data (labeled as "Exp. R\&A" in Fig. 3). Fig. 3 shows that this rough model predicts a local minimum of the damping, although both the 
position of $\delta_{A}^{+}$and the value of the local minimum are incorrectly predicted. Apparently, the reflection by the turbulence is overestimated in the rigid plate model; in addition, a phase should be added to the reflected wave due to the elastic properties of the turbulence, ${ }^{2,28}$ but this is excluded in the rigid plate model since $\phi=0$. Peters et al. ${ }^{3}$ related the phase shift to the "memory" effects of the turbulence, i.e, the fact that the plate does not react instantly to the incident wave, but after a time delay. This corresponds to a relaxation process due to non-equilibrium, and the relaxation time of the turbulence $t_{\tau}$ which characterizes the time delay, was chosen to be $t_{\tau}=100 \nu / u_{\tau}^{2}$. Peters et al. ${ }^{3}$ pointed out that the measured damping of the sound wave appears to approach the local minimum if the time period of the sound wave coincides with the relaxation time, since then $\omega^{+}=2 \pi / 100 \approx 0.06$ is of the same order of 0.014 (remember that $\delta_{A}^{+}=\sqrt{2 / \omega^{+}} \approx 12$ if $\omega^{+}=0.014$ ). If the relaxation time $t_{\tau}$ is used, the phase shift becomes $\phi=\omega t_{\tau}=100 \omega^{+}$; the value for $\delta_{\nu}$ was adjusted to 12.5 in the extended rigid plate model to fit the data of Ronneberger and Ahrens ("Exp. R\&A"). Fig. 3 shows that this extended rigid plate model can successfully predict the local minimum of the damping in the "Exp. R\&A" data; it also shows good agreement with the measured imaginary part of the wall shear stress impedance when $10<\delta_{A}^{+}<17$.

Howe ${ }^{13}$ included the concept of relaxation time in the modeling and proposed a more physical model compared to the rigid plate approximation. The model assumes that the turbulent flow is modulated by the sound waves so, for the first order approximation, the eddy-viscosity $\nu_{T}$ is frequency dependent. This is done by utilizing the same idea that has been used in the rigid plate model, that the vorticity modal fields are entirely uninfluenced by turbulent diffusions within the viscous sublayer $\delta_{\nu}$; while outside the sublayer an eddy-viscosity which is linearly dependent on the distance from the wall is used to model the turbulent flow. Since the thickness of the acoustic boundary $l_{\text {vor }}$ increases as the frequency decreases, the thickness of the viscous sublayer $\delta_{\nu}$ should accordingly decrease to emphasize the turbulent diffusions over the viscous effects. The eddy-viscosity then can be written as

$$
\nu_{T}=\left\{\begin{array}{ll}
0, & x_{2}<\delta_{\nu}(\omega) \\
\kappa u_{\tau}\left[x_{2}-\delta_{\nu}(\omega)\right], & x_{2}>\delta_{\nu}(\omega)
\end{array},\right.
$$

and the effective viscous sublayer $\delta_{\nu}(\omega)$ is determined by an empirical formula

$$
\delta_{\nu}=\frac{6.5 \nu}{u_{\tau}}\left[1+\frac{1.7\left(\omega t_{\tau}\right)^{3}}{1+\left(\omega t_{\tau}\right)^{3}}\right],
$$

where the relaxation time $t_{\tau}$ is involved. The parameters 6.5 and 1.7 were chosen to fit the local minimum in the data of Peters et al. ${ }^{3}$ (labeled as "Exp. Peters et al." in Fig. 3). Based on Eq. (33), Howe ${ }^{13}$ derived the following equation for the normalized wall shear stress by setting $\omega t_{\tau}=100 \omega^{+}$

$$
Z_{\tau}=\operatorname{conj}\left\{F_{A}\left(\frac{1}{\kappa} \sqrt{\mathrm{i} \omega^{+}}, 6.5\left[1+\frac{1.7\left(100 \omega^{+}\right)^{3}}{1+\left(100 \omega^{+}\right)^{3}}\right] \sqrt{\mathrm{i} \omega^{+}}\right)\right\},
$$

where "conj" denotes the complex conjugate, and $F_{A}$ is defined as

$$
F_{A}(a, b)=\frac{\mathrm{i}\left[H_{1}^{(1)}(a) \cos b-H_{0}^{(1)}(a) \sin b\right]}{H_{0}^{(1)}(a) \cos b+H_{1}^{(1)}(a) \sin b},
$$

and $H_{0}^{(1)}$ and $H_{1}^{(1)}(a)$ are the Hankel functions of the zeroth and the first order. Although Howe's model fits the damping data better than other models when $\delta_{A}^{+}>15$, as shown in Fig. 3, it does not give better predictions of the imaginary part of $Z_{\tau}$, compared to the rigid plate model proposed by Peters et al.. ${ }^{3}$ However, Howe's model is less "crude" than the rigid plate models, since it implements a finite value of the eddy-viscosity outside the viscous sublayer $\delta_{\nu}$; besides, Howe's model has the merit of being continuous in the entire duct cross-section, so it can be implemented in more complex perturbation equations, e.g., including mean flow convections. ${ }^{15}$

When it comes to the non-equilibrium effects, both Howe's model, Eq. (35), and the rigid plate model proposed by Peters et al. show the importance of such effects in sound-turbulent interactions ${ }^{\text {IT }}$; nevertheless,

$\overline{\boldsymbol{\top}}$ Mao and Hanratty ${ }^{14}$ also included the non-equilibrium effects in their relaxation model, but it is complicated to implement in the calculations here; besides, it is also based on empirical formulas, so the details of this model is not introduced in this paper. 
neither of these empirical models has demonstrated on a theoretical ground how such effects participate in the interactions. Eq. (33) also shows that Howe's model does not incorporate the elastic properties of the turbulence, as there is no phase shift between the stress and the wave shear strain rate.

The present authors recently proposed a model ${ }^{17}$ based on the phase and time averaged dynamic equations of the Reynolds stress. By applying the quasi-linear assumption to the Reynolds stress equations, ${ }^{29} \mathrm{a}$ relaxation equation for the perturbation Reynolds stress $\tilde{r}_{12}$ is derived

$$
\frac{\overline{\mathrm{D}} \tilde{r}_{12}}{\overline{\mathrm{D}} t}=-\frac{1}{t_{\tau}} \tilde{r}_{12}-\frac{2 \nu_{T}}{t_{\tau}} \frac{\partial \tilde{u}_{1}}{\partial x_{2}}
$$

where $\overline{\mathrm{D}} / \overline{\mathrm{D}} t=\partial / \partial t+\bar{u}_{1} \partial / \partial x_{1}$ for a parallel mean flow. The eddy-viscosity $\nu_{T}$ is the one in the quasi-static state, e.g., the Cess' formula Eq. (27). By setting the time derivative to $\overline{\mathrm{D}} \tilde{r}_{12} / \overline{\mathrm{D}} t=0$, Eq. (37) reduces to the quasi-static model, Eq. (10). This time derivative is retained here in order to account for the nonequilibrium effects, the turbulent relaxation time $t_{\tau}$ is therefore involved, and $t_{\tau}=160 \nu / u_{\tau}$ was chosen to fit the local minimum in the data (both "Exp. R\&A" and "Exp. Peters et al."). For low Mach number cases, we omit the convection term $\bar{u}_{1} \partial / \partial x_{1}$, and Eq. (37) has the solution in the frequency domain

$$
\tilde{r}_{12}=\frac{-2 \nu_{T}}{1+\mathrm{i} \omega t_{\tau}} \frac{\partial \tilde{u}_{1}}{\partial x_{2}} .
$$

Compared to the quasi-static model, Eq. (38) shows an equivalent eddy-viscosity $\nu_{A}$ due to the acoustic perturbation, which is ${ }^{* * *}$

$$
\nu_{A}=\frac{2 \nu_{T}}{1+\mathrm{i} \omega t_{\tau}},
$$

and this perturbation eddy-viscosity adds a phase shift of $-\arctan \omega t_{\tau}$, ranging from zero towards $-\pi / 2$, to the resulting stress $\tilde{r}_{12}$. This phase shift, as has been discussed earlier, is due to the non-equilibrium effects, the visco-elastic properties of the turbulence during the interaction process is therefore included in this model, Eq. (39). Eq. (39) shows that $\nu_{A} \sim 2 \nu_{T}$ as $\omega t^{*} \rightarrow 0$, this is in accordance with the fact that sound-turbulent interactions approach a quasi-static state for low frequency sound waves. The zero frequency limit corresponds to the case when $\delta_{A}^{+} \rightarrow \infty$, but in Fig. 3 the measured wave damping tends to asymptotically approach the quasi-static state from $\delta_{A}^{+} \approx 23$. On the other hand, $\left|\nu_{A}\right| \sim 0$ as $\omega t^{*} \rightarrow \infty$, which is the quasi-laminar model (with a phase shift $-\arctan \omega t_{\tau}$ approaching $-\pi / 2$ ). As has been discussed earlier, the sound-turbulent interactions can be described by the quasi-laminar model in the high frequency limit, and it is proved by the measurements as shown in Fig. 3, i.e., $Z_{\tau} \sim 1+\mathrm{i}$ as $\delta_{A}^{+} \rightarrow \infty$.

The wall shear stress impedance can be computed from this non-equilibrium model, Eq. (39), by replacing the effective eddy-viscosity $\nu_{e}^{+}$in Eq. (28) with

$$
\nu_{e}^{+}=1+\nu_{A}^{+}=1+\frac{2 \nu_{T}^{+}}{1+160 \mathrm{i} \omega^{+}},
$$

where Cess' formula, (27), is still used for $\nu_{T}^{+}$.

The computed results are plotted in Fig. 3, and we can see that this non-equilibrium model, as derive from a relaxation equation, can predict a local minimum in the damping, and the predicted value locates between those from the two set of measured data. Besides, the measured imaginary part of $Z_{\tau}$ from both measurements displays a slight increase when $\delta_{A}^{+}>23$. The existence of this increase had been doubted by Ronneberger and Ahrens, ${ }^{2}$ as the measuring of the imaginary part of $Z_{\tau}$ is very sensitive to the errors in the Mach number. This increase is, however, predicted by both Howe's model and the non-equilibrium model, and the one predicted by the latter model shows better agreement with the data by Peters et al.. ${ }^{3}$

\section{Sound propagation in fully developed turbulent pipe flow}

Experimental data of the sound attenuation in fully developed turbulent pipe flow are available (see Tab. 1 for the details of the experiments). The attenuation is computed from the experimentally determined wave numbers, $k$, measured at different Mach numbers. In this section experimental data is used to verify the

\footnotetext{
${ }^{* * *}$ This relation should be $\nu_{A}=\nu_{T} /\left(1+\mathrm{i} \omega t_{\tau}\right)$ if one following the modeling in Reynolds and Hussain, ${ }^{6}$ where the quasi-static model is $\tilde{r}_{12}=\nu_{T} \partial \tilde{u}_{1} / \partial x_{2}$.
} 
non-equilibrium model Eq. (38). Firstly the perturbation equations including mean flow convection terms are given, and, in addition to the low Mach number approximation Eq. (38), a model for $\tilde{r}_{i j}$ with convection is derived. The computed results are then compared with the experimental data, and discussions based on the results are given.

\begin{tabular}{|c|c|c|c|}
\hline Authors & Pipe diameter $(\mathrm{m})$ & Frequency $(\mathrm{Hz})$ & Mach number $\left[U_{b} / c_{0}\right]$ \\
\hline Peters et $a l .{ }^{3}$ & 0.030 & 88 & $0.018-0.10$ \\
\hline Allam and bom ${ }^{4}$ & 0.035 & 100 & $0.01-0.22$ \\
\hline Allam and bom ${ }^{4}$ & 0.035 & 250 & $0.01-0.22$ \\
\hline Ronneberger and Ahrens ${ }^{2}$ & 0.020 & 630 & $0.01-0.33$ \\
\hline
\end{tabular}

Table 1. Data for the measurements.

\section{III.A. Governing equations}

To begin with, the axis-symmetric perturbation equations in the frequency domain are given in Eqs. (41) to (44), which can be derived from the Navier-Stokes equations by using the triple decomposition Eq. (5). In cylindrical coordinates, this can be written as ${ }^{15,17,30,31}$

$$
\begin{gathered}
\int_{0}^{2 \pi} \int_{0}^{R}\left[\left(\mathrm{i} \omega+\bar{u}_{x} \frac{\partial}{\partial x}\right) \tilde{\rho}+\rho_{0} \frac{\partial \tilde{u}_{x}}{\partial x}\right] r \mathrm{~d} r=0 \\
\left(\mathrm{i} \omega+\bar{u}_{x} \frac{\partial}{\partial x}\right) \tilde{u}_{x}+\frac{1}{\rho_{0}} \frac{\partial \widetilde{p}}{\partial x}-\nu\left(\frac{\partial^{2} \tilde{u}_{x}}{\partial r^{2}}+\frac{1}{r} \frac{\partial \tilde{u}_{x}}{\partial r}\right)-\frac{\partial \tilde{r}_{x r}}{\partial r}=0 \\
\frac{\partial \tilde{p}}{\partial r}=0 \\
\left(\mathrm{i} \omega+\bar{u}_{x} \frac{\partial}{\partial x}\right) \tilde{T}-\frac{1}{\rho_{0} C_{p}}\left(\mathrm{i} \omega+\bar{u}_{x} \frac{\partial}{\partial x}\right) \tilde{p}-\chi\left(\frac{\partial^{2}}{\partial r^{2}}+\frac{1}{r} \frac{\partial}{\partial r}\right) \tilde{T}-\frac{\partial \tilde{q}_{r}}{\partial r}=0,
\end{gathered}
$$

where

$$
\tilde{q}_{r}=\left\langle T^{\prime} u_{r}^{\prime}\right\rangle-\overline{T^{\prime} u_{r}^{\prime}},
$$

is the wave induced perturbation from the background turbulent heat flux. The governing equation of $\tilde{r}_{x r}$ is given in Eq. (38), which is coupled with the wave quantity $\tilde{u}_{x}$ here. The boundary layer approximation, i.e., that the radial velocity is much smaller than the axial velocity, and that the radial changes are much larger than changes in the axial direction, is herein made. The integral form of the continuity equation, Eq. (41), is used in order to eliminate the transverse velocity components without any approximation. The ideal gas law also provides $\tilde{p} / p_{0}=\tilde{\rho} / \rho_{0}+\tilde{T} / T_{0}$, where $p_{0}$ and $T_{0}$ are the steady pressure and temperature that are used here as reference values. Eq. (41) can then be written as:

$$
\int_{0}^{2 \pi} \int_{0}^{R}\left[\left(\mathrm{i} \omega+\bar{u}_{x} \frac{\partial}{\partial x}\right)\left(\frac{\tilde{p}}{p_{0}}-\frac{\tilde{T}}{T_{0}}\right)+\frac{\partial \tilde{u}_{x}}{\partial x}\right] r \mathrm{~d} r=0 .
$$

The mean velocity $\bar{u}_{x}$ can be solved from the Reynolds-averaged mean flow equation with the Boussinesq eddy-viscosity assumptions ${ }^{12}$

$$
\left(\frac{1}{r}+\frac{\mathrm{d}}{\mathrm{d} r}\right)\left[\left(\nu+\nu_{T}\right) \frac{\mathrm{d} \bar{u}_{x}}{\mathrm{~d} r}\right]=\frac{1}{\rho_{0}} \frac{\mathrm{d} \bar{p}}{\mathrm{~d} x},
$$

where Cess' formula, Eq. (27), is used for $\nu_{T}$.

On the pipe wall, no-slip and iso-termal boundary conditions are assumed; and symmetric boundary conditions are applied at the center of the pipe.

If we use the form

$$
\tilde{F}=\hat{\tilde{F}}(r) e^{\mathrm{i}\left(\omega t-k_{0} \Gamma x\right)}
$$


as the temporal and the spatial dependancy for the wave quantities in Eqs. (42) to (46), we then have an eigenvalue problem. The eigenvalue, $\Gamma$, is the complex non-dimensional wave number, whose imaginary part denotes the wave attenuation, while the real part denotes the phase shift. The reference wave number is defined as $k_{0}=\omega / c_{0}$.

By neglecting the convection effects in Eq. (37), an effective eddy-viscosity $\nu_{T}$, Eq. (39), for the sound wave field is derived. This allows us to use the gradient-diffusion hypothesis for the perturbation heat flux $\tilde{q}_{r}$, i.e. ${ }^{13,15,17}$

$$
\tilde{q}_{r}=\rho_{0} c_{p} \alpha_{A} \frac{\partial \tilde{T}}{\partial r}
$$

where $\alpha_{A}=\nu_{A} / \operatorname{Pr}_{T}$ is the corresponding thermal diffusivity for the sound wave, and $\operatorname{Pr}_{T}=0.7$ is the turbulent Prandtl number in air, and is assumed to be constant.

If the mean flow convection term is included in Eq. (37), the $x$-direction variation of $\tilde{r}_{x r}$ should be considered. An intrinsic assumption is that $\tilde{r}_{x r}$ has the same $x$-direction dependency as in Eq. (48), so Eq. (37) can be written as

$$
\mathrm{i} \omega\left(1-M_{x} \Gamma\right) \tilde{r}_{x r}=-\frac{1}{t_{\tau}} \tilde{r}_{x r}-\frac{2 \nu_{T}}{t_{\tau}} \frac{\partial \tilde{u}_{x}}{\partial r},
$$

where $M_{x}=\bar{u}_{x} / c_{0}$ is the local Mach number in the stream-wise direction. In analogy to Eq. (38), the perturbation Reynolds stress is written as

$$
\tilde{r}_{x r}=\nu_{A} \frac{\partial \tilde{u}_{x}}{\partial r}
$$

where $\nu_{A}$ is the perturbation eddy-viscosity with convection effects included, which is

$$
\nu_{A}=\frac{2 \nu_{T}}{1+\mathrm{i} \omega t_{\tau}\left(1-\Gamma M_{x}\right)} .
$$

Applying the Taylor expansion of $\nu_{A}$ centered at $M_{x}=0$, we have

$$
\nu_{A}=\frac{2 \nu_{T}}{1+\mathrm{i} \omega t_{\tau}}\left[1+\frac{\mathrm{i} \omega t_{\tau}}{1+\mathrm{i} \omega t_{\tau}} \Gamma M_{x}+\frac{\left(\mathrm{i} \omega t_{\tau}\right)^{2}}{\left(1+\mathrm{i} \omega t_{\tau}\right)^{2}}\left(\Gamma M_{x}\right)^{2}\right]+\mathcal{O}\left(\mathrm{M}^{3}\right),
$$

where the mean flow Mach number $\mathrm{M}$ is defined from $M_{x}$ as

$$
\mathrm{M}=\frac{\int_{0}^{R} 2 \pi M_{x} r \mathrm{~d} r}{\pi R^{2}}
$$

The zeroth order term of $M_{x}$ on the right hand side of Eq. (53) is exactly the same as Eq. (39), indicating that Eq. (39) is the zeroth order approximation for small Mach numbers. For the first/second order approximations, we can retain the terms up to the first/second order of $M_{x}$ on the right hand side of Eq. (53), and neglect the higher order terms.

\section{III.B. Results and Discussion}

In this section we present the comparisons of the non-equilibrium model, i.e., Eqs. (51) and (52), with the available measurement data of the wave attenuation (see Tab. 1).

The zeroth order, Eq. (39), the first order,

$$
\nu_{A}=\frac{2 \nu_{T}}{1+\mathrm{i} \omega t_{\tau}}\left(1+\frac{\mathrm{i} \omega t_{\tau}}{1+\mathrm{i} \omega t_{\tau}} \Gamma M_{x}\right)
$$

and the second order,

$$
\nu_{A}=\frac{2 \nu_{T}}{1+\mathrm{i} \omega t_{\tau}}\left[1+\frac{\mathrm{i} \omega t_{\tau}}{1+\mathrm{i} \omega t_{\tau}} \Gamma M_{x}+\frac{\left(\mathrm{i} \omega t_{\tau}\right)^{2}}{\left(1+\mathrm{i} \omega t_{\tau}\right)^{2}}\left(\Gamma M_{x}\right)^{2}\right],
$$

approximations for the perturbation eddy-viscosity are implemented in the eigenvalue problem, and the computed attenuations are compared with the experimental data in Fig. 4. The attenuation $\alpha_{ \pm}$is obtained 
from the imaginary part of the computed $k_{0} \Gamma$. The attenuation is normalized by the damping in a quiescent fluid $\alpha_{0}$, which is computed from the imaginary part of $k$ in Eq. (23).

The variation of the attenuation with the acoustic boundary layer thickness is similar to that of the real part of the $Z_{\tau}$ (see Fig. 3), i.e., local minimums are also observed in the attenuation. Due to the mean convection effects, the attenuations of the up- and down-stream propagating waves are different, namely the attenuation of the up-stream propagating wave is larger than the attenuation of the down-stream propagating wave. Since the convections are considered in the perturbation equations (42) to (46), two of the computed eigenvalues are supposed to represent the up- and down-stream propagating waves respectively. These two eigenvalues are chosen from the two least attenuated ones, i.e., eigenvalues with the smallest imaginary part. ${ }^{15,30}$

The spectral method is used here to solve the eigenvalue problem. Details of the numerical schemes are documented in Weng et al.. ${ }^{17}$

From Fig. 4 we can see that the predicted results with the zeroth order non-equilibrium model, Eq. (39), show very good accordance with the experimental data. In comparison, the first order model, Eq. (55), and the second order model, Eq. (56), show less good results. For low Mach number flows, the first and higher order terms of $M_{x}$ are expected to be negligible. It is therefore surprising to see in Fig. 4 that the differences between the first and the second order models can barely be observed, while the differences between the zeroth and the first order models are considerable. The reason might be the involvement of the convection of the wave shear strain rate in $\tilde{r}_{x r}$ when higher order models are used. For the first order model, for instance, this can be seen by inserting Eq. (53) into Eq. (51), yielding

$$
\begin{aligned}
\tilde{r}_{x r} & =\frac{2 \nu_{T}}{1+\mathrm{i} \omega t_{\tau}} \frac{\partial \tilde{u}_{x}}{\partial r}+\frac{2 \nu_{T}}{\left(1+\mathrm{i} \omega t_{\tau}\right)^{2}}\left(\mathrm{i} \omega t_{\tau} \Gamma M_{x}\right) \frac{\partial \tilde{u}_{x}}{\partial r} \\
& =\frac{2 \nu_{T}}{1+\mathrm{i} \omega t_{\tau}} \frac{\partial \tilde{u}_{x}}{\partial r}-\frac{2 \nu_{T} t_{\tau}}{\left(1+\mathrm{i} \omega t_{\tau}\right)^{2}}\left(\bar{u}_{x} \frac{\partial^{2} \tilde{u}_{x}}{\partial r \partial x}\right)
\end{aligned} .
$$

Equation (57) shows that the perturbation Reynolds stress does not only depend on the local wave shear strain rate $\partial \tilde{u}_{x} / \partial r$, but also the convection of it by the mean flow, due to the involvement of the higher order model. This indicates that the higher order models differ from the eddy-viscosity model which only depends on the local shear strain rate. Consequently the eddy-diffusivity model, Eq. (49), which is based on the eddy-viscosity assumption, might not be valid in this case. We have tried to used the first order model for $\tilde{r}_{i j}$, while keeping using the zeroth order model for $\tilde{q}_{r}$, in the calculation of the eigenvalue problem, then the computed results showed little differences from the results computed with the zeroth order model that has been shown in Fig. 4. This somehow verifies the invalidity of the eddy-diffusivity model if higher order models are used. As a consequence, more complex models, other than the eddy-diffusivity model, Eq. (49), may be required if one seeks to use higher order models for $\tilde{r}_{i j}$.

In addition, the failure of the higher order models may also be caused by unbalanced terms in the perturbation equation (44): in the perturbation eddy-viscosity Eqs. (55) and (56), the higher order terms in $M_{x}$ are involved. This may require those terms which have been neglected under the low Mach number assumptions to be included in Eq. (44), or the equation is unbalanced if higher order models are used. However, Eq. (65) (see "Appendix") shows that it is very difficult to model these neglected terms. So if the simplified perturbation equation (44) is used, the zeroth order approximation for the perturbation eddy-viscosity, Eq. (52), should also be used. 
(a): Peters et al. (1993)

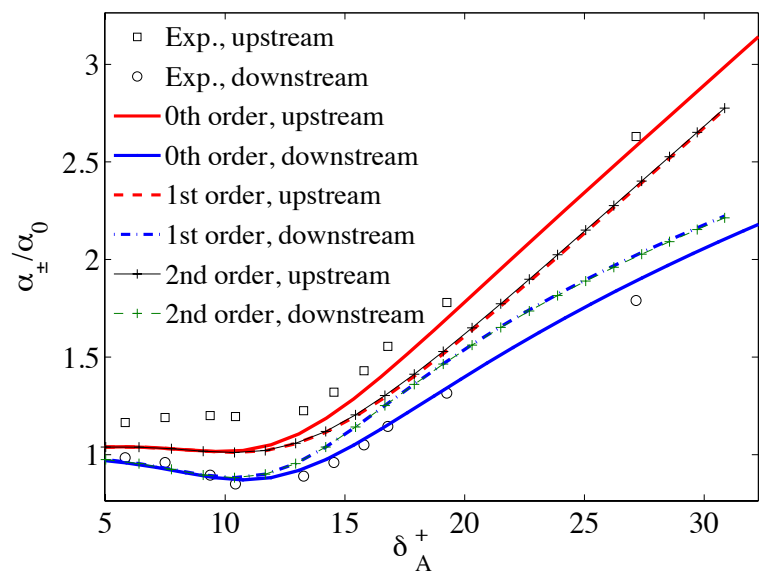

(c): Allam et al. (2006)

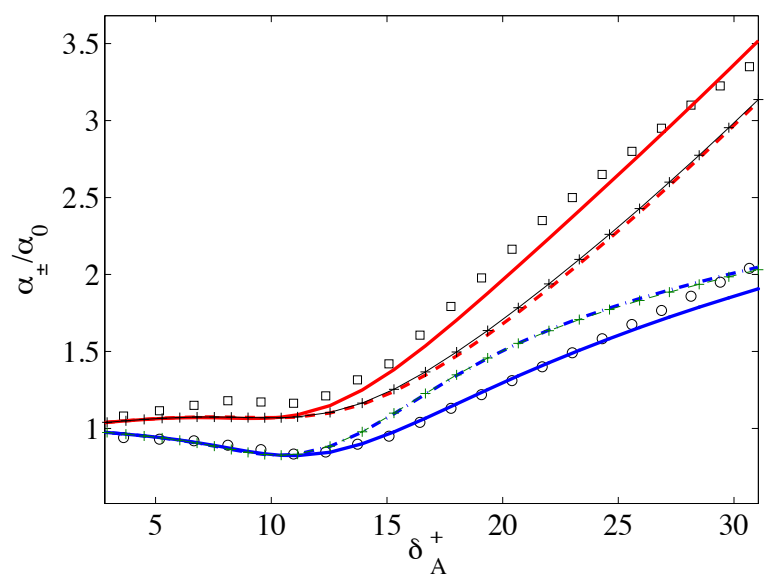

(b): Allam et al. (2006)

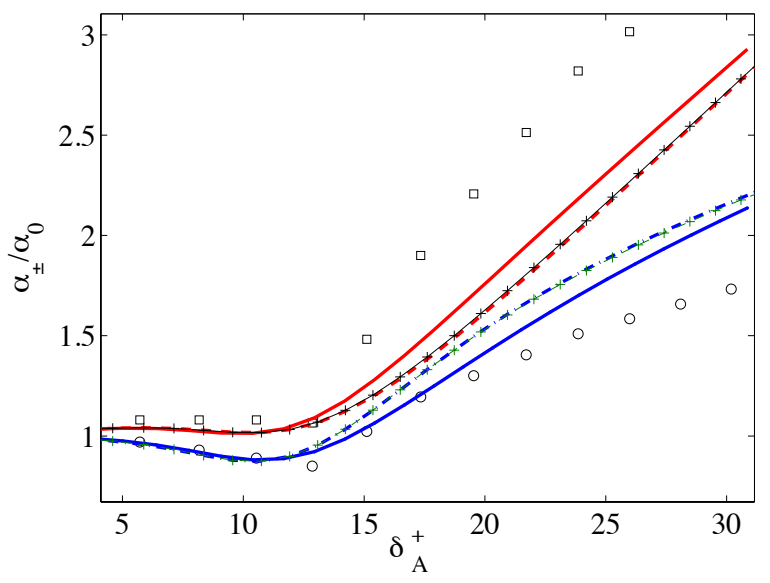

(d): Ronneberger and Ahrens (1977)

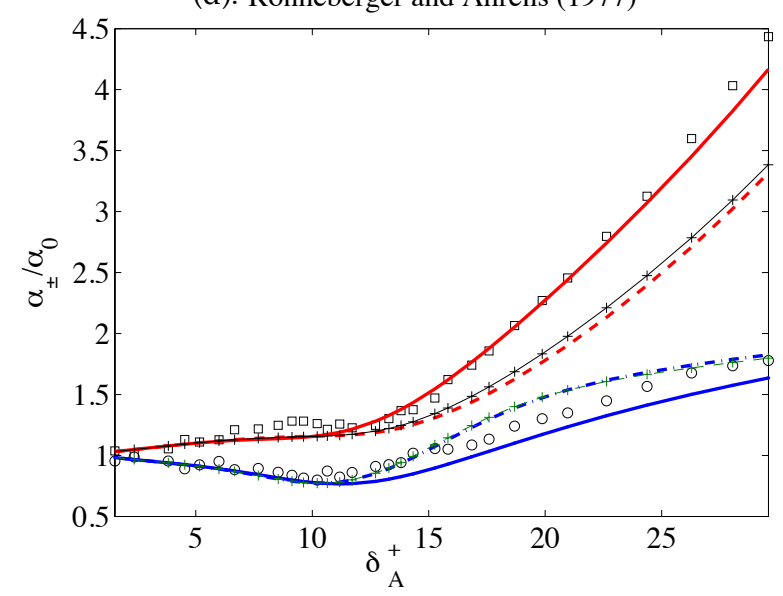

Figure 4. Attenuations of up- and down-stream waves. The experimental data are from a) Peters et al., ${ }^{3}$ $f=88 \mathbf{H z}$; b) Allam and bom, ${ }^{4} f=100 \mathbf{H z}$; c) Allam and bom, ${ }^{4} f=250 \mathbf{H z}$; Ronneberger and Ahrens, ${ }^{2} f=630$ Hz. The computed results are obtained by using the $0^{\text {th }}$ order (Eq. (39)), the $1^{\text {st }}$ order (Eq. (55)), and the $2^{\text {nd }}$ order (Eq. (56)) perturbation eddy-viscosity model separately.

\section{Conclusion}

An analytical non-equilibrium model ${ }^{17}$ for the perturbation Reynolds stress has been applied for prediction of the wall shear stress impedance of sound waves propagating in low Mach number turbulent duct flows. Compared to previous successful models, ${ }^{2,3,13}$ this non-equilibrium model also shows satisfactory agreement with the experimental data. Meanwhile, it provides a clearer interpretation of the sound-turbulence interactions process, that the turbulence acts as visco-elastic fluid to the sound wave, i.e. the turbulent stress does not only depend on the present wave shear strain rate, but also has the "memory" of its past.

In addition, the mean flow convection effect has been included in the non-equilibrium model. The resulting first order, Eq. (55), and second order, Eq. (56), approximations for the perturbation Reynolds stress, together with its zeroth order approximation (without the convection effects), Eq. (39), have been applied to compute the wave attenuation and the results are compared with experimental data. The comparisons show that the zeroth order approximation of the perturbation Reynolds stress gives much better agreement with the measured data than the higher order approximations. Conclusion drawn from this observation is that one may have to introduce more complicated models for the perturbation heat flux, or to include the neglected terms in the perturbation equations, in order to correctly implement the higher order approximations. 


\section{Appendix: Derivation of the temperature perturbation equation}

Conservation of energy (for ideal gas with constant specific heats) gives ${ }^{32}$

$$
\frac{\partial T}{\partial t}+u_{j} \frac{\partial T}{\partial x_{j}}=\frac{1}{c_{p} \rho}\left(\frac{\partial p}{\partial t}+u_{j} \frac{\partial p}{\partial x_{j}}\right)+\frac{\Phi}{c_{p} \rho}+\alpha_{t h} \frac{\partial^{2} T}{\partial x_{j} \partial x_{j}},
$$

where the dissipation $\Phi$ is defined by

$$
\Phi=\mu\left[\frac{\partial u_{i}}{\partial x_{j}} \cdot \frac{\partial u_{i}}{\partial x_{j}}+\frac{\partial u_{j}}{\partial x_{i}} \cdot \frac{\partial u_{i}}{\partial x_{j}}-\frac{2}{3}\left(\frac{\partial u_{i}}{\partial x_{i}}\right)^{2}\right]
$$

In this study we consider low Mach number flow, the Boussinesq approximation ${ }^{32,33}$ is herein employed, namely the density variations are small enough to be neglected for the turbulent flow field. The propagation of the sound, however, relies on the density variations, but these acoustic fluctuations are assumed undetected by the turbulent flow. Thus the following relations for the mean and turbulent fluctuation velocities derived under Boussinesq approximation still hold:

$$
\frac{\partial \bar{u}_{i}}{\partial x_{i}}=\frac{\partial u_{i}^{\prime}}{\partial x_{i}}=0
$$

The decomposition of the total density field accordingly becomes

$$
\rho(\boldsymbol{x}, t)=\rho_{0}+\widetilde{\rho}(\boldsymbol{x}, t)
$$

where $\rho_{0}$ is the density at ambient state.

Applying the triple decomposition Eq. (5) to the temperature field, $T$, and using the relations given in Eqs. (60) and (61), Eq. (58) becomes

$$
\begin{aligned}
& \frac{\partial\left(\widetilde{T}+T^{\prime}\right)}{\partial t}+\left(\bar{u}_{j}+\widetilde{u}_{j}+u_{j}^{\prime}\right) \frac{\partial\left(\bar{T}+\widetilde{T}+T^{\prime}\right)}{\partial x_{j}} \\
= & \frac{1}{c_{p} \rho_{0}}\left[\frac{\partial\left(\widetilde{p}+p^{\prime}\right)}{\partial t}+\left(\bar{u}_{j}+\widetilde{u}_{j}+u_{j}^{\prime}\right) \frac{\partial\left(\bar{p}+\widetilde{p}+p^{\prime}\right)}{\partial x_{j}}\right] \\
& +\frac{\nu}{c_{p}}\left[\frac{\partial\left(\bar{u}_{i}+\widetilde{u}_{i}+u_{i}^{\prime}\right)}{\partial x_{j}} \cdot \frac{\partial\left(\bar{u}_{i}+\widetilde{u}_{i}+u_{i}^{\prime}\right)}{\partial x_{j}}+\frac{\partial\left(\bar{u}_{j}+\widetilde{u}_{j}+u_{j}^{\prime}\right)}{\partial x_{i}} \cdot \frac{\partial\left(\bar{u}_{i}+\widetilde{u}_{i}+u_{i}^{\prime}\right)}{\partial x_{j}}\right. \\
& \left.-\frac{2}{3} \frac{\partial \widetilde{u}_{i}}{\partial x_{i}} \cdot \frac{\partial \widetilde{u}_{j}}{\partial x_{j}}\right]+\alpha_{t h} \frac{\partial^{2}\left(\bar{T}+\widetilde{T}+T^{\prime}\right)}{\partial x_{j} \partial x_{j}} .
\end{aligned}
$$

Time averaging Eq. (62) gives

$$
\begin{aligned}
& \bar{u}_{j} \frac{\partial \bar{T}}{\partial x_{j}}+\overline{\widetilde{u}_{j} \frac{\partial \widetilde{T}}{\partial x_{j}}}+\frac{\partial \overline{T^{\prime} u_{j}^{\prime}}}{\partial x_{j}} \\
= & \frac{1}{c_{p} \rho_{0}}\left(\bar{u}_{j} \frac{\partial \bar{p}}{\partial x_{j}}+\overline{\widetilde{u}_{j} \frac{\partial \widetilde{p}}{\partial x_{j}}}+\frac{\partial \overline{p^{\prime} u_{j}^{\prime}}}{\partial x_{j}}\right) \\
& +\frac{\nu}{c_{p}}\left(\frac{\partial \bar{u}_{i}}{\partial x_{j}} \cdot \frac{\partial \bar{u}_{i}}{\partial x_{j}}+\overline{\frac{\partial u_{i}^{\prime}}{\partial x_{j}}} \cdot \frac{\partial u_{i}^{\prime}}{\partial x_{j}}+\frac{\partial \widetilde{u}_{i}}{\partial x_{j}} \cdot \frac{\partial \widetilde{u}_{i}}{\partial x_{j}}+\frac{\partial \bar{u}_{j}}{\partial x_{i}} \cdot \frac{\partial \bar{u}_{i}}{\partial x_{j}}\right. \\
& \left.+\frac{\partial u_{j}^{\prime}}{\partial x_{i}} \cdot \frac{\partial u_{i}^{\prime}}{\partial x_{j}}+\frac{\overline{\partial \widetilde{u}_{j}}}{\partial x_{i}} \cdot \frac{\partial \widetilde{u}_{i}}{\partial x_{j}}-\frac{2}{3} \frac{\partial \widetilde{u}_{i}}{\partial x_{i}} \cdot \frac{\partial \widetilde{u}_{j}}{\partial x_{j}}\right)+\alpha_{t h} \frac{\partial^{2} \bar{T}}{\partial x_{j} \partial x_{j}}
\end{aligned}
$$


Phase averaging Eq. (62) gives

$$
\begin{aligned}
& \frac{\partial \widetilde{T}}{\partial t}+\left(\bar{u}_{j}+\widetilde{u}_{j}\right) \frac{\partial(\bar{T}+\widetilde{T})}{\partial x_{j}}+\frac{\partial\left\langle T^{\prime} u_{j}^{\prime}\right\rangle}{\partial x_{j}} \\
= & \frac{1}{c_{p} \rho_{0}}\left[\frac{\partial \widetilde{p}}{\partial t}+\left(\bar{u}_{j}+\widetilde{u}_{j}\right) \frac{\partial(\bar{p}+\widetilde{p})}{\partial x_{j}}+\frac{\partial\left\langle p^{\prime} u_{j}^{\prime}\right\rangle}{\partial x_{j}}\right] \\
& +\frac{\nu}{c_{p}}\left(\frac{\partial \bar{u}_{i}}{\partial x_{j}} \cdot \frac{\partial \bar{u}_{i}}{\partial x_{j}}+2 \frac{\partial \bar{u}_{i}}{\partial x_{j}} \cdot \frac{\partial \widetilde{u}_{i}}{\partial x_{j}}+\frac{\partial \widetilde{u}_{i}}{\partial x_{j}} \cdot \frac{\partial \widetilde{u}_{i}}{\partial x_{j}}+\left\langle\frac{\partial u_{i}^{\prime}}{\partial x_{j}} \cdot \frac{\partial u_{i}^{\prime}}{\partial x_{j}}\right\rangle\right. \\
& +\frac{\partial \bar{u}_{j}}{\partial x_{i}} \cdot \frac{\partial \bar{u}_{i}}{\partial x_{j}}+2 \frac{\partial \widetilde{u}_{j}}{\partial x_{i}} \cdot \frac{\partial \bar{u}_{i}}{\partial x_{j}}+\frac{\partial \widetilde{u}_{j}}{\partial x_{i}} \cdot \frac{\partial \widetilde{u}_{i}}{\partial x_{j}}+\left\langle\frac{\partial u_{j}^{\prime}}{\partial x_{i}} \cdot \frac{\partial u_{i}^{\prime}}{\partial x_{j}}\right\rangle \\
& \left.-\frac{2}{3} \frac{\partial \widetilde{u}_{i}}{\partial x_{i}} \cdot \frac{\partial \widetilde{u}_{j}}{\partial x_{j}}\right)+\alpha_{t h} \frac{\partial^{2}(\bar{T}+\widetilde{T})}{\partial x_{j} \partial x_{j}} .
\end{aligned}
$$

Subtracting (63) from (64), and applying the linearization approximation, we get

$$
\begin{aligned}
& \frac{\partial \widetilde{T}}{\partial t}+\widetilde{u}_{j} \frac{\partial \bar{T}}{\partial x_{j}}+\bar{u}_{j} \frac{\partial \widetilde{T}}{\partial x_{j}} \\
= & \frac{1}{c_{p} \rho_{0}}\left(\frac{\partial \widetilde{p}}{\partial t}+\bar{u}_{j} \frac{\partial \widetilde{p}}{\partial x_{j}}+\widetilde{u}_{j} \frac{\partial \bar{p}}{\partial x_{j}}\right) \\
& +\frac{2 \nu}{c_{p}}\left(\frac{\partial \bar{u}_{i}}{\partial x_{j}} \cdot \frac{\partial \widetilde{u}_{i}}{\partial x_{j}}+\frac{\partial \widetilde{u}_{j}}{\partial x_{i}} \cdot \frac{\partial \bar{u}_{i}}{\partial x_{j}}\right)+\alpha_{t h} \frac{\partial^{2} \widetilde{T}}{\partial x_{j} \partial x_{j}} \\
& -\frac{\partial\left(\left\langle T^{\prime} u_{j}^{\prime}\right\rangle-\overline{T^{\prime} u_{j}^{\prime}}\right)}{\partial x_{j}}+\frac{1}{c_{p} \rho_{0}} \frac{\partial\left(\left\langle p^{\prime} u_{j}^{\prime}\right\rangle-\overline{p^{\prime} u_{j}^{\prime}}\right)}{\partial x_{j}} \\
& +\frac{\nu}{c_{p}}\left(\left\langle\frac{\partial u_{i}^{\prime}}{\partial x_{j}} \cdot \frac{\partial u_{i}^{\prime}}{\partial x_{j}}\right\rangle-\frac{\partial u_{i}^{\prime}}{\partial x_{j}} \cdot\left\langle\frac{\partial u_{j}^{\prime}}{\partial x_{i}} \cdot \frac{\partial u_{i}^{\prime}}{\partial x_{j}}\right\rangle-\frac{\partial u_{j}^{\prime}}{\partial x_{i}} \cdot \frac{\partial u_{i}^{\prime}}{\partial x_{j}}\right) .
\end{aligned}
$$

By assuming constant values of mean temperature, $\bar{T}$, and pressure, $\bar{p}$, one can neglect the terms $\widetilde{u}_{j} \partial \bar{T} / \partial x_{j}$ and $\widetilde{u}_{j} \partial \bar{p} / \partial x_{j}$ in Eq. (65). However, there are still more unknowns in Eq. (65), compared to Eq. (44). In the Boussinesq approximation, the pressure term, $\partial p / \partial t+u_{j} \partial p / \partial x_{j}$, and the dissipation term, $\Phi$, are neglected for low Mach numbers, ${ }^{33}$ so the modeling of the term $\left\langle p^{\prime} u_{j}^{\prime}\right\rangle-\overline{p^{\prime} u_{j}^{\prime}}$ and the dissipation terms in Eq. (65) can be avoided. If the term $\partial \tilde{p} / \partial t+\tilde{u}_{j} \partial \tilde{p} / \partial x_{j}$ in Eq. (65) is retained to account for the perturbation pressure change, ${ }^{15,30}$ one can derive the simplified perturbation equation (44) for the temperature field.

\section{Acknowledgments}

This work was funded by VR (the Swedish Research Council) and by the Linn Flow Centre at KTH.

\section{References}

\footnotetext{
1991.

${ }^{1}$ Pierce, A. D., Acoustics : an introduction to its physical principles and applications, the Acoustical Society of America,

${ }^{2}$ Ronneberger, D. and Ahrens, C. D., "Wall shear stress caused by small amplitude perturbations of turbulent boundarylayer flow - An experimental investigation," Journal of Fluid Mechanics, Vol. 83, Dec. 1977, pp. 433-464.

${ }^{3}$ Peters, M. C. A. M., Hirschberg, A., Reijnen, A. J., and Wijnands, A. P. J., "Damping and reflection coefficient measurements for an open pipe at low Mach and low Helmholtz numbers," Journal of Fluid Mechanics, Vol. 256, 1993, pp. 499-534.

${ }^{4}$ Allam, S. and Åbom, M., "Investigation of damping and radiation using full plane wave decomposition in ducts," Journal of Sound and Vibration, Vol. 292, No. 3-5, 2006, pp. 519-534.
} 
${ }^{5}$ Comte, P., Haberkorn, M., Bouchet, G., Pagneux, V., and Aurégan, Y., "Large-Eddy Simulation of acoustic propagation in a turbulent channel flow," Direct and Large-Eddy Simulation VI, edited by E. Lamballais, R. Friedrich, B. Geurts, and O. Métais, Springer Netherlands, 2006, pp. 521-528.

${ }^{6}$ Reynolds, W. C. and Hussain, A. K. M. F., "The mechanics of an organized wave in turbulent shear flow. Part 3. Theoretical models and comparisons with experiments," Journal of Fluid Mechanics, Vol. 54, No. 02, 1972, pp. $263-288$.

${ }^{7}$ Hussain, A. K. M. F. and Reynolds, W. C., "The mechanics of an organized wave in turbulent shear flow," Journal of Fluid Mechanics, Vol. 41, 1970, pp. 241-258.

${ }^{8}$ Hussain, A. K. M. F. and Reynolds, W. C., "The mechanics of an organized wave in turbulent shear flow. Part 2. Experimental results," Journal of Fluid Mechanics, Vol. 54, No. 02, 1972, pp. 241-261.

${ }^{9}$ Ingard, U. and Singhal, V. K., "Sound attenuation in turbulent pipe flow," The Journal of the Acoustical Society of America, Vol. 55, No. 3, 1974, pp. 535-538.

${ }^{10}$ Howe, M. S., "The interaction of sound with low Mach number wall turbulence, with application to sound propagation in turbulent pipe flow," Journal of Fluid Mechanics, Vol. 94, No. 04, 1979, pp. 729-744.

${ }^{11}$ Howe, M. S., "On the Absorption of Sound by Turbulence and Other Hydrodynamic Flows," IMA Journal of Applied Mathematics, Vol. 32, No. 1-3, 1984, pp. 187-209.

${ }^{12}$ Pope, S. B., Turbulent flows, Cambridge University Press, 2000.

${ }^{13}$ Howe, M. S., "The damping of sound by wall turbulent shear layers," The Journal of the Acoustical Society of America, Vol. 98, No. 3, 1995, pp. 1723-1730.

${ }^{14}$ Mao, Z. X. and Hanratty, T. J., "Studies of the wall shear stress in a turbulent pulsating pipe flow," Journal of Fluid Mechanics, Vol. 170, 1986, pp. 545-564.

${ }^{15}$ Knutsson, M. and Åbom, M., "The effect of turbulence damping on acoustic wave propagation in tubes," Journal of Sound and Vibration, Vol. 329, No. 22, 2010, pp. $4719-4739$.

${ }^{16}$ Dokumaci, E., "On attenuation of plane sound waves in turbulent mean flow," Journal of Sound and Vibration, Vol. 320, No. 4-5, 2009, pp. $1131-1136$.

${ }^{17}$ Weng, C., Boij, S., and Hanifi, A., "The attenuation of sound by turbulence in internal flows," The Journal of the Acoustical Society of America, in press, Manuscript accepted for publication.

${ }^{18}$ Kierkegaard, A., Boij, S., and Efraimsson, G., "A frequency domain linearized Navier-Stokes equations approach to acoustic propagation in flow ducts with sharp edges," The Journal of the Acoustical Society of America, Vol. 127, No. 2, 2010, pp. $710-719$.

${ }^{19}$ Tijdeman, H., "On the propagation of sound waves in cylindrical tubes," Journal of Sound and Vibration, Vol. 39, No. 1, 1975 , pp. $1-33$. 2649 .

${ }^{20}$ Daly, B. J. and Harlow, F. H., "Transport Equations in Turbulence," Physics of Fluids, Vol. 13, No. 11, 1970, pp. 2634-

${ }^{21}$ Mankbadi, R. R. and Liu, J. T. C., "Near-wall response in turbulent shear flows subjected to imposed unsteadiness," Journal of Fluid Mechanics, Vol. 238, 1992, pp. 55-71.

${ }^{22}$ Cess, R. D., "A survey of the literature on heat transfer in turbulent tube flow." Tech. Rep. 8-0529-R24, Westinghouse Research Report, 1958.

${ }^{23}$ Reynolds, W. C. and Tiederman, W. G., "Stability of turbulent channel flow, with application to Malkus's theory," Journal of Fluid Mechanics, Vol. 27, No. 02, 1967, pp. 253-272.

${ }^{24}$ McConaghy, G. A. and Hanratty, T. J., "Influence of drag reducing polymers on turbulent mass transfer to a pipe wall," AIChE Journal, Vol. 23, No. 4, 1977, pp. 493-500.

${ }^{25}$ Willis, A. P., Hwang, Y., and Cossu, C., "Optimally amplified large-scale streaks and drag reduction in turbulent pipe flow," Phys. Rev. E, Vol. 82, Sep 2010, pp. 036321.

${ }^{26}$ McKeon, B. J., LI, J., Jiang, W., Morrison, J. F., and Smits, A. J., "Further observations on the mean velocity distribution in fully developed pipe flow," Journal of Fluid Mechanics, Vol. 501, 2004, pp. 135-147.

${ }^{27}$ Boyd, J. P., Chebyshev and Fourier Spectral Methods, 2nd edition, Dover Pubns, 2001.

${ }^{28}$ Davis, R. E., "Perturbed turbulent flow, eddy viscosity and the generation of turbulent stresses," Journal of Fluid Mechanics, Vol. 63, No. 04, 1974, pp. 673-693.

${ }^{29}$ Hamlington, P. E. and Dahm, W. J. A., "Reynolds stress closure for nonequilibrium effects in turbulent flows," Physics of Fluids, Vol. 20, No. 11, 2008.

${ }^{30}$ Astley, R. J. and Cummings, A., "Wave propagation in catalytic converters: formulation of the problem and finite element to solution scheme," Journal of Sound and Vibration, Vol. 188, No. 5, 1995, pp. 635 - 657.

${ }^{31}$ Peat, K. S., "A First Approximation To The Effects Of Mean Flow On Sound Propagation Through Cylindrical Capillary Tubes," Journal of Sound and Vibration, Vol. 175, No. 4, 1994, pp. 475 - 489.

${ }^{32}$ Batchelor, G. K., An introduction to fluid dynamics, Cambridge mathematical library, Cambridge University Press, 2000.

${ }^{33}$ Kundu, P. K. and Cohen, I. M., Fluid mechanics, No. v. 10 in Fluid mechanics, Academic Press, 2008. 